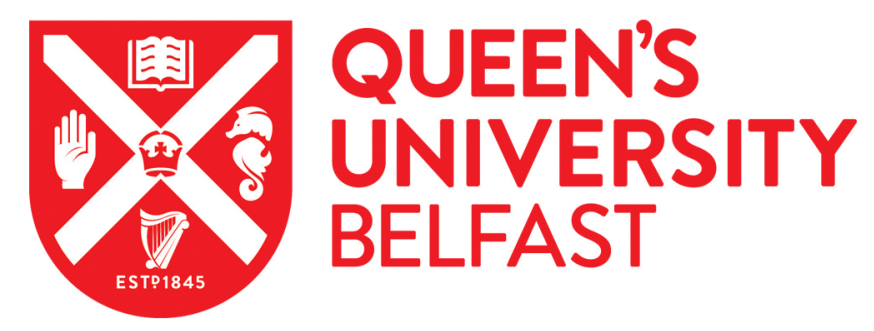

\title{
Evidential event inference in transport video surveillance
}

Hong, X., Huang, Y., Ma, W., Varadarajan, S., Miller, P., Liu, W., Romero, M. J. S., Martinez del Rincon, J., \& Zhou, H. (2016). Evidential event inference in transport video surveillance. Computer Vision and Image Understanding, 144, 276-297. https://doi.org/10.1016/j.cviu.2015.10.017

Published in:

Computer Vision and Image Understanding

Document Version:

Peer reviewed version

Queen's University Belfast - Research Portal:

Link to publication record in Queen's University Belfast Research Portal

Publisher rights

(c) 2016 Elsevier Ltd. This manuscript version is made available under the CC-BY-NC-ND 4.0 license http://creativecommons.org/licenses/bync-nd/4.0/which permits distribution and reproduction for non-commercial purposes, provided the author and source are cited.

\section{General rights}

Copyright for the publications made accessible via the Queen's University Belfast Research Portal is retained by the author(s) and / or other copyright owners and it is a condition of accessing these publications that users recognise and abide by the legal requirements associated with these rights.

Take down policy

The Research Portal is Queen's institutional repository that provides access to Queen's research output. Every effort has been made to ensure that content in the Research Portal does not infringe any person's rights, or applicable UK laws. If you discover content in the Research Portal that you believe breaches copyright or violates any law, please contact openaccess@qub.ac.uk. 


\title{
Evidential Event Inference in Transport Video Surveillance
}

\author{
Xin Hong ${ }^{\mathrm{a}, *}$, Yan Huang ${ }^{\mathrm{a}}$, Wenjun $\mathrm{Ma}^{\mathrm{b}}$, Sriram Varadarajan ${ }^{\mathrm{a}}$, Paul Miller ${ }^{\mathrm{a}}$, \\ Weiru Liu ${ }^{a}$, Maria Jose Santofimia Romero ${ }^{c}$, Jesus Martinez del Rincon ${ }^{\mathrm{a}}$, Huiyu Zhou ${ }^{\mathrm{a}}$ \\ ${ }^{a}$ Centre for Secure Information Technologies, School of EEECS, Queen's University Belfast, BT3 9DT, UK \\ ${ }^{b}$ Department of Philosophy, East China Normal University, Shanghai, China \\ ${ }^{c}$ Computer Architecture and Networks Group, University of Castilla-La Mancha, Spain
}

\begin{abstract}
This paper presents a new framework for multi-subject event inference in surveillance video, where measurements produced by low-level vision analytics usually are noisy, incomplete or incorrect. Our goal is to infer the composite events undertaken by each subject from noise observations. To achieve this, we consider the temporal characteristics of event relations and propose a method to correctly associate the detected events with individual subjects. The Dempster-Shafer (DS) theory of belief functions is used to infer events of interest from the results of our vision analytics and to measure conflicts occurring during the event association. Our system is evaluated against a number of videos that present passenger behaviours on a public transport platform namely buses at different levels of complexity. The experimental results demonstrate that by reasoning with spatio-temporal correlations, the proposed method achieves a satisfying performance when associating atomic events and recognising composite events involving multiple subjects in dynamic environments. Keywords: Transport surveillance, video events, event modelling, reasoning under uncertainty, spatio-temporal constraint, minimum conflict optimisation, event association and recognition
\end{abstract}

\section{Introduction}

Security information and event management systems (SIEMs) are well-established within the field of network security. Physical SIEMs are also well-established within the physical security domain. However, many of the events that they deal with are of a very simple nature with

\footnotetext{
*Corresponding author

Email address: x.hong@qub.ac.uk (Xin Hong)
} 
a high degree of certainty, e.g., intrusion alarms, access control. Intelligent analysis and correlation/aggregation of incoming events from different sources represents a challenge to these systems.

Recent developments in the field of video analytics have resulted in a new source of events for PSEIM that can provide rich semantically meaningful information with regard to situational awareness. However, unlike earlier event types, these can have a degree of uncertainty and can conflict with one another. Whilst the video analytics community has been making progress on generating low-level events, typically termed action recognition [? ? ? , little thought has been given to how one manages events of this nature over a period of time to give higher-level composite events [? ]. However, as this technology has started to migrate from the laboratory to the commercial sector, there is a growing realisation of the need to manage the events generated by video analysis software. By manage we mean the representation, storage, reasoning and mining of events.

One of the main tasks of event management systems is that of event composition, whereby patterns of events across a distributed network are detected. Event composition allows us to represent different events and also to instantly infer events of interest by applying rules to combine existing events. In addition, new situations can be captured by simply adding a new rule instead of modifying custom code, hence ensuring a flexible solution for evolving situations. Event composition can either be deterministic, or probabilistic, or both [? ? ], however, to date only a few researchers have addressed the problems of imperfect information, or information from different sources that may be conflicting.

For the past decade or so, the deployment of CCTV in major urban centres and cities has become well established. Recently, CCTV technology has begun to be deployed on public transport systems such as buses and trains. The application domain of interest to us is the analysis of people's behaviour as they move into, remain in, and move out of seated areas. Whilst this scenario has received very little attention to date within the computer vision community, seated areas are ubiquitous in many application scenarios. For example, these can be found onboard transport platforms such as buses, trains and planes. They are also to be found in many transport hubs such as train stations and departure lounges in airports. Other sectors where they are to be found include sports stadiums, entertainment venues such as concert halls, and leisure venues such as restau- 
rants and bars. Of particular interest to us is the bus scenario and detecting anti-social or criminal behaviour on buses. Studies have shown that the vast majority of crime carried out on transport platforms such as buses is by young males [? ]. Therefore, having knowledge of the gender of passengers, and how they are moving relative to one another, as well as their seated positions, enables one to infer the degree of threat and likelihood of an anti-social/criminal incident occurring. The vast majority of events on a bus consist of passengers undertaking normal journeys in which nothing untoward happens. This can be decomposed as: a passenger boarding the bus at an entrance, moving into the saloon area along the gangway, and taking a seat, which we classify as atomic events. Similarly, when exiting, a passenger stands, moves along the gangway towards an exit, and then disembarks from the bus. We classify both these as composite events. Less regular composite events include a passenger changing a seat whilst the bus is moving. This could indicate that one of the passengers is either being intimidated or threatened by another passenger.

Unfortunately, imperfect information frequently occurs in real world applications. For example, in the case of a person entering the bus doorway, the person may be classified as male with a certainty of $85 \%$ by the classification analytics, however, the remainder does not imply that the person is female with a $15 \%$ certainty, rather, it is unknown. Hence, it can only give imperfect information for the remaining $15 \%$. Imperfect information is usually caused by the unreliability of the information sources. For example, in the classification example above, the camera may have been tampered with, illumination could be poor, or the classifier training set may be unrepresentative. Any or all of these can result in imperfect information which cannot be represented by probability measures.

In this work, we investigate the use of evidential reasoning, for dealing with low-level, or atomic events that are uncertain, and combining them into higher level composite events that have semantic meaning from a security viewpoint. Our main contributions can be summarised as follows:

A. The development of a novel technique for associating identities with atomic events.

B. One of the first attempts at integrating video analytics with an event reasoning framework.

C. First demonstration of the recognition of composite, semantically meaningful, events on- 
board the challenging environment of a moving transport platform (bus).

The rest of this paper is organised as follows. In Section 2, we review related work. Section 3 provides a preliminary treatment of the Dempster-Shafer theory of evidence and temporal relation representation. We propose a new framework of subject-event association and composite event recognition in Section 4, a case study in Section Appendix B illustrates how our framework works. In Section 5, the experimental methodology is described and results are presented. Finally, Section 6 concludes this paper and discusses the future work.

\section{Related Work}

During the recent past there has been an extensive amount of work on video action recognition by the computer vision research community ([? ? ] and references therein). However, most of this work has been on what we call atomic event recognition, e.g. running, walking etc, which have a unique and enclosed, but limited, semantic meaning in relation to the application context. Less emphasis has been given to the use of reasoning for aggregating atomic events so that high-level semantically rich composite events can be recognised. This straddles the boundary between the computer vision and artificial intelligence communities, and perhaps is the reason why there has been less work in this area [? ]. In this section we first review vision-based action recognition and then event reasoning approaches for composite event recognition.

\subsection{Action Recognition}

Given a specific scenario, where interactive elements are known, simple action recognition can be performed by applying human detection to video sequences, and from these generate trajectories which can then be used to describe the actions of the detected subjects. For example, part-based techniques can be used to locate [?] and track [? ] human body parts. These trajectories can then be modelled using methods such as Hidden Markov Models (HMMs) [? ].

Although these techniques are simple to implement and effective for simple actions and scenarios, they fail to provide richer information of the sort needed to recognise more subtle actions. Extending this methodology to estimate the trajectory of the human pose [? ? ], i.e. the trajectory of each body part, allows this. However, current methods have been shown not to be robust for real 
scenarios and multiple actors [? ]. It is also debatable whether such fine grain detail is really necessary for action recognition [? ]. In most practical scenarios human detection and pose estimation can be difficult, due to the presence of background clutter and foreground occlusions. Therefore, another approach is to treat a sequence, or part thereof, as a single entity from which low-level spatio-temporal features can be extracted and classified as belonging to a particular action. For example, Klaser et al. [? ] proposed calculating the 3D Histogram of Oriented Gradients (HoG) over a space-time volume in order to characterise actions. Similarly, Ke et al. generated over segmented spatio-temporal volumes and optical flow correlation and then used a distance metric to determine the subset of spatio-temporal volumes that best matched a parts-based event template [? ]. A common approach is to describe a video sequence as an unordered set of space-time features, e.g., bag of visual words (BoV). Wang et al. proposed a BoV approach to describe videos by dense trajectories [? ]. Oneata et al. applied the Fisher vector representation, an extension of BoV, to action classification [? ]. By employing approximations to Fisher normalisations they obtained a speed-up of an order of magnitude whilst maintaining state-of-the-art action recognition performance. In a different approach to BoV, Sadanand and Corso proposed the use of action banks, consisting of a bank of individual template-based action detectors that provided location features by maximum poling of volumetric correlation outputs [? ]. The resulting feature vectors generated for different actions and scales were then concatenated and used to train an SVM for classification.

An event scenario that has received considerable attention is the detection of abandoned bags. Tian et al. applied the results of background subtraction for detecting static and foreground regions and, using a novel segmentation algorithm, the former were then classified as being abandoned or removed [? ]. Human detection and tracking are also employed in order to reduce false positives. In other work [? ], Fan et al. proposed representing abandoned objects alerts by relative attributes, e.g., staticness, foregroundness and abandonment. A ranking function, learnt using low-level spatial and temporal features, was used to determine the relative strengths of these attributes. Their system outperformed other state-of-the-art techniques in terms of precision for the PETS2006 and AVSS-AB datasets.

Another scenario that has been extensively investigated is that of crowd analysis for security and/or safety purposes. As has been noted in [? ], techniques developed for non-crowded scenarios 
tend to fail in crowded scenes. As such, research has focused on addressing those issues, e.g., occlusion and complex collective behaviours, unique to crowds. Idrees et al. proposed identifying prominent individuals within a crowd that are relatively easy to track, and then using the concept of neighbourhood motion occurrence to determine the behaviour of individuals within the crowd [? ]. Zhou et al. presented a new mixture model of dynamic pedestrian agents to learn collective behaviour patterns of pedestrians in crowded scenes [? ]. In [? ], Yi et al. developed a technique for detecting stationary foreground regions by applying sparse constraints along spatial and temporal dimensions to produce a 3D stationary map. This is then used to detect four types of stationary group behaviours; gathering, relocating, joining and dispersing.

All of the previous work reviewed thus far, assumed prior knowledge of the actions, i.e., they were pre-defined. A more complex problem is the detection of unknown or unusual actions. Leach et al reported on an unsupervised context-aware approach which takes into account scene and social contexts to detect anomalous behaviour [? ]. Static and dynamic agents were used by Cho and Kang to model individual and group behaviours as a BoVs [? ]. Kittler et al. surveyed the area of anomaly detection and proposed the use of context for anomaly detection in video sequences [? ].

\subsection{Event Reasoning}

In this section we review related work on composite event recognition and reasoning, which is the focus of the work reported herein. Composite events have greater semantic meaning to endusers than atomic events, and are high-level semantic interpretations from a set of atomic events [? ]. They are not easily identifiable using image features, but, rather, by recognition of their composing events [?].

There are two major approaches to composite event recognition, classification and inference. In the former, one approach is to use actions, scenes and objects as semantic attributes for their classification. Chen et al. [?] started with the identification of candidate concepts for an event by firstly crawling Flickr to search for images with tags related to keywords in the event description. WordNet was then used to filter out noisy tags and each concept verified based on the visual cohesiveness of the images associated with it. This was followed by building a concept visual 
model using a Support Vector Machine classifier. They found that their approach outperformed others based on low-level visual features for a supervised event modelling task. Li et al. [? ] proposed decomposition of a video sequence into short-term segments, which were modelled by a dictionary of attribute dynamics templates using a binary dynamic system. It is common to see probabilistic approaches applied to the recognition of atomic events, due to their limited ability of modelling interrelations between events in both space and time, and representing structural information in event composition.

Inference-based approaches to composite event recognition usually involve development of an event modelling and reasoning mechanism. Composite events consist of a set of atomic events that occur over a considerable time-span and that may have a partial ordering or be concurrent. Thus, one of the main AI-based approaches to composite event recognition is to infer them by reasoning about atomic events. Works on visual event modelling and reasoning tend to follow two major trends; declarative and probabilistic.

In declarative approaches, descriptive templates are used to model events, such as context-free grammar [? ] and Petri-Nets [? ]. Ryoo and Aggarwal [? ] used context-free grammar to model interactions of primitive actions and to recognise composite activities for multi-subject scenarios. Petri-Nets are used as a formalism to model complex logical temporal and spatial relations in event composition [? ]. These are derived from semantic descriptions of events in video event ontology languages such as VERL. Recently, ontologies, a semantic web technique, have been used to automate the representation of composite events [? ]. In [? ], an event ontology for representing complex spatio-temporal events by a composition of simpler ones was proposed. The hierarchy includes primitive events, single-thread composite events and multi-thread composite events. Inferences are made in a bottom-up fashion. Declarative approaches work satisfactorily when describing event semantics. However, major drawbacks include an inability to handle multiple subjects and fragility to uncertainty in sensor measurements, which frequently exist in real applications.

In probabilistic approaches, such as HMMs [? ], Dynamic Bayesian Networks [? ], and multi-agent methods [? ? ], models are constructed to represent events. While these demonstrate impressive robustness to uncertainty in video analytics, they do not define semantically meaning- 
ful sub-events. Thus, it is not easy to describe the composition of an event at a semantic level. Though DBNs are more general than HMMs, by considering dependencies between several random variables, the temporal model is still usually Markovian, as is the case for HMMs [? ]. Their models can only handle sequential activities and fail to describe complex relations between subevents. Consequently, they often lack flexibility; hence it is difficult to apply them to dynamic problems in real applications [? ].

Recently hybrid approaches have emerged that combine declarative and probabilistic properties. These tend to combine the rich representation ability of declarative approaches with the uncertainty reasoning mechanism of probabilistic approaches. Stochastic grammars [? ] have been used for parking lot surveillance in [? ]. Tran et al. [? ] applied Markov logic networks (MLNs) to probabilistically infer events in video surveillance where noise and missing observations are serious problems. First-order logic production rules are used to represent common sense domain knowledge. A weight is associated to each rule to indicate their confidence. In [? ], Kanaujia et al. also proposed the use of MLNs for recognising complex events over a sensor network consisting of four cameras. In their approach, rather than use a single Markov network (MN) for representing all activities, they explicitly partitioned the MN into multiple activity specific networks. They addressed the issue of uncertainty, due to the noisy sensor data and video analytic errors, by generating predicates with an associated probability. Semantic information extracted at each level from the lowest level visual processing is propagated to sub-event detection by each MLN engine and then to a higher-level complex event module to recognise complex events. To tackle the problem of recognising coordinated events in challenging videos with cluttered background and occlusion, Brendel et al. [? ] proposed the formulation of probabilistic event logic (PEL) for representing temporal constraints among events. Lavee et al. [? ] introduced a certainty score to Petri Nets to cope with uncertain event observations.

Though the majority of previous declarative and probabilistic approaches have been applied to single subject scenarios, a few have tackled the more difficult problem of event recognition in multi-subject videos. Among them, attention has focused on recognising events from understanding the interactions between subjects. These works presume that low-level video analytics can provide sufficient information for the detection of simple semantic events, which often appears 
untrue in real world applications.

The approach proposed in this paper fits in the hybrid group and focuses on multi-subject video applications. Our solution is different from previous hybrid approaches in several ways. Firstly, we adopt the Dempster-Shafer (DS) theory of evidence [? ? ] to handle uncertainty in event recognition, from observations, to event detection and inference. Imperfect information frequently occurs in real world applications. For example, in bus surveillance, when a person enters the bus the camera detects a face and classifies it as female with a certainty of $75 \%$. However, the remainder does not imply that the face is male with a $25 \%$ certainty, rather it is deemed to be unknown because the gender classification analysis does not have enough information to distribute the remaining $25 \%$ to male or female. In contrast, with probability theory such information can only be represented as $p($ female $) \geq 0.75$ and $p($ male $) \leq 0.25$, which is difficult to use for reasoning. Furthermore, the propagation and combination mechanisms of DS theory are superior for composing complex events from simple sub-events and atomic events detected from noisy observations. Hierarchical network templates are used to model the structural semantics of complex event composition. Similar to [? ] , we use Allen's temporal interval relation modelling [? ] to represent temporal relations between events; however, we go further and deduce the association of events with different subjects in a multi-subject scene. One of challenges for event recognition in multi-subject videos is that video analytics often results in errors, such as missed detections, and broken tracks due to occlusion. To address this, we develop constraint rules, using the temporal relationships between events, and use conflict factors of Dempster's combination rule to measure conflict in event combinations, enabling us to associate events to a particular subject. Part of the current manuscript has been published in conference proceedings [50, 51].

\section{Preliminaries}

In this section, we introduce the main concepts of reasoning under uncertainty and temporal relation representation, which we have relied upon in developing our proposed approach. 


\subsection{Dempster-Shafer Theory of Evidence}

The fundamental technique of evidential reasoning that this work uses is the Dempster-Shafer theory of evidence (DS theory), which originated from Dempster's work [? ] and further extended by Shafer [? ]. DS theory is a generalisation of traditional probability theory and describes the propositional space of possible situations for a given problem by a finite, non-empty set called the frame of discernment, denoted as $\Theta$. Uncertainty related to propositions of the problem is represented by a mass function over the power set $2^{\Theta}$ : the set of all subsets of $\Theta$.

Definition 1. The mapping $2^{\Theta} \rightarrow[0,1]$ is a basic belief assignment, also called a mass function $m$, satisfying: (1) $m(\emptyset)=0$; (2) $\sum_{A \subseteq \Theta} m(A)=1$.

A mass value can be committed to a subset, $A$, of $\Theta$ with either single or multiple elements. All $A$ are called focal elements if $m(A)>0$, where $m(A)$ is attributed to $A$ and only $A$. Due to lack of information this mass value cannot be further distributed amongst specific elements in $A$, which makes mass functions different with probability functions. When $m(\Theta)=1$ and $m(A)=0$ for all $A \neq \Theta$, the mass function represents total ignorance, called a vacuous mass function. When all focal elements of a mass function are singletons, the mass function is reduced to a probability function.

When two frames of discernment $\Theta_{G}$ and $\Theta_{H}$ hold relations described by an evidential mapping $\Gamma^{*}$, the mass function occurring on $\Theta_{G}$ can be projected to $\Theta_{H}$ via $\Gamma^{*}$ as follows [? ].

$$
m_{\Theta_{H}}\left(H_{j}\right)=\sum_{i} m_{\Theta_{G}}\left(g_{i}\right) f\left(g_{i} \rightarrow H_{j}\right)
$$

$\Gamma^{*}: \Theta_{G} \rightarrow 2^{2^{\Theta_{H} \times[0,1]}}$ assigns an element $g_{i} \in \Theta_{G}$ to a set of subset-mass pairs in the following way:

$$
\Gamma^{*}\left(g_{i}\right)=\left(\left(H_{i 1}, f\left(g_{i} \rightarrow H_{i 1}\right)\right), \ldots,\left(H_{i m}, f\left(g_{i} \rightarrow H_{i m}\right)\right)\right)
$$

where $H_{i j} \subseteq \Theta_{H}, i=1, \ldots, n, j=1, \ldots, m$, and $f: \Theta_{G} \times \Theta_{H} \rightarrow[0,1]$ satisfying (a) $H_{i j} \neq \emptyset$, $j=1, \ldots, m$; (b) $\sum_{j=1}^{m} f\left(g_{i} \rightarrow H_{i j}\right)=1$; (c) $\Gamma^{*}\left(\Theta_{G}\right)=\left(\left(\Theta_{H}, 1\right)\right)$.

When all the $f\left(g_{i} \rightarrow H_{i j}\right)$ are either 1 or 0 , an evidential mapping $\Gamma^{*}$ becomes a multi-valued mapping $\Gamma: \Theta_{G} \rightarrow 2^{\Theta_{H}}$. A mass function from frame $\Theta_{G}$ can be translated to frame $\Theta_{H}$ as [?]:

$$
m\left(H_{j}\right)=\sum_{\Gamma\left(g_{i}\right)=H_{j}} m\left(g_{i}\right)
$$


where $g_{i} \in \Theta_{G}, H_{j} \subseteq \Theta_{H}$.

One advantage of DS theory is that it provides a mechanism of aggregating multiple pieces of evidence from different sources. When mass functions $m_{1}$ and $m_{2}$ are obtained from two independent sources over the same frame of discernment $\Theta$, the consensus mass function $m$ can be obtained by fusing them using Dempster's rule of combination as follows.

$$
m(C)=(1-k)^{-1} \sum_{A \cap B=C} m_{1}(A) m_{2}(B)
$$

where $k=\sum_{A \cap B=\emptyset} m_{1}(A) m_{2}(B) \neq 1$, is considered to be a conflict factor that numerically measures the degree of conflict between two pieces of evidence. When $k=0$, two pieces of evidence are completely consistent. When $k=1$, the two are completely inconsistent. The combination rule is both commutative and associative.

It is common that information provided by a source may not be completely credible. To reflect the reliability of the source, a discount rate $r \in[0,1]$ is introduced in [? ]. The original mass function $m$ from a source is discounted:

$$
m^{r}(A)= \begin{cases}(1-r) m(A), & A \subset \Theta \\ r+(1-r) m(\Theta), & A=\Theta .\end{cases}
$$

For decision making, Smets [?] proposed the pignistic transformation of mass functions.

Definition 2. Assume that there exists mass function $m(A), A \subseteq \Theta$. For every element $g$ of $\Theta$, the pignistic probability, denoted BetP, can be calculated:

$$
\operatorname{BetP}(g)=\sum_{g \in A} \frac{m(A)}{|A|}
$$

where $|A|$ is the number of elements of $\Theta$ in $A$.

The pignistic probability is the DS counterpart of the subjective probability that would quantify the agent's beliefs according to the Bayesians [? ].

\subsection{Temporal Relations}

Allen proposed a method for modelling temporal relations in [? ? that enables the representation of multiple subjects' actions over a period of time extending from the present to the 
future. In Allen's model, temporal information is represented by intervals than points. In this way, real-world events taking place over a time interval can be handled within the same modelling framework as instantaneous events, by treating the latter as occurring over a time interval with the same start and end time. The time of an event can be relative to a reference point rather than being absolute. To describe temporal correlations between two event instances that take place within two time intervals respectively, Allen defined thirteen relations as depicted in Table 1.

Table 1: The Allen's thirteen interval temporal relations on $I X=\left[I X_{a}, I X_{b}\right]$ and $I Y=\left[I Y_{a}, I Y_{b}\right]$

\begin{tabular}{|l|l|l|l|}
\hline Interval relation & Symbol & Inverse & Endpoint relations \\
\hline$I X$ before $I Y$ & $\mathrm{~b}$ & $\mathrm{a}$ & $I X_{a}<I Y_{a}, I X_{a}<I Y_{b}, I X_{b}<I Y_{a}, I X_{b}<I Y_{b}$ \\
\hline$I X$ meets $I Y$ & $\mathrm{~m}$ & $\mathrm{mi}$ & $I X_{a}<I Y_{a}, I X_{a}<I Y_{b}, I X_{b}=I Y_{a}, I X_{b}<I Y_{b}$ \\
\hline$I X$ overlaps $I Y$ & $\mathrm{o}$ & $\mathrm{oi}$ & $I X_{a}<I Y_{a}, I X_{a}<I Y_{b}, I X_{b}>I Y_{a}, I X_{b}<I Y_{b}$ \\
\hline$I X$ starts $I Y$ & $\mathrm{~s}$ & $\mathrm{si}$ & $I X_{a}=I Y_{a}, I X_{a}<I Y_{b}, I X_{b}>I Y_{a}, I X_{b}<I Y_{b}$ \\
\hline$I X$ during $I Y$ & $\mathrm{~d}$ & $\mathrm{di}$ & $I X_{a}>I Y_{a}, I X_{a}<I Y_{b}, I X_{b}>I Y_{a}, I X_{b}<I Y_{b}$ \\
\hline$I X$ finishes $I Y$ & $\mathrm{f}$ & $\mathrm{fi}$ & $I X_{a}>I Y_{a}, I X_{a}<I Y_{b}, I X_{b}>I Y_{a}, I X_{b}=I Y_{b}$ \\
\hline$I X$ equal $I Y$ & eq & eq & $I X_{a}=I Y_{a}, I X_{a}<I Y_{b}, I X_{b}>I Y_{a}, I X_{b}=I Y_{b}$ \\
\hline
\end{tabular}

Allen's temporal model will allow us to enforce constraints in our event inference for both continuous and discrete events.

\section{METHODOLOGY}

This section describes our system for uncertain atomic event management from multiple sensors and composite event inference. The system is proposed in the context of video-surveillance for public transport platforms.

\subsection{System Outline}

The main purpose of video surveillance is to provide situational awareness of a specific place over a period of time. In this context, therefore, an event is an observation (or collection of observations) that has semantic meaning. An event can be simple or complex depending on the level of relevant semantic information provided. To distinguish these two different concepts, we 


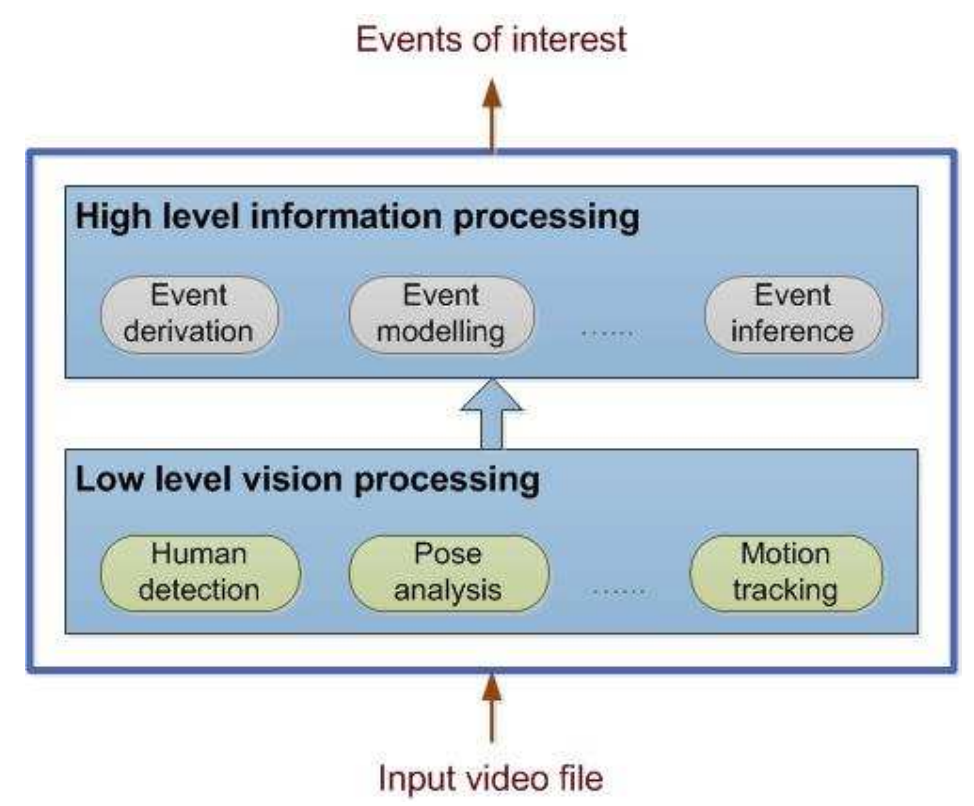

Figure 1: System of intelligent event management for video surveillance

call the former an atomic event and the latter a composite event. An atomic event can be directly detected using video analytics and/or sensors. Atomic events can then be aggregated to generate composite events which are more semantically meaningful.

Our system is composed of two main stages, shown in Fig. 1, and integrates computer vision techniques with knowledge representation and reasoning mechanisms. In the first stage, human subjects are detected and video analytics are then generated in order to provide low-level semantic components such as "a female face has been detected" and "a person has moved from the door towards the gang-way". The second stage is designed to recognise significant events based on a semantic hierarchy obtained from domain knowledge. At this level, the events of interest are recognised based on the information derived at the lower-level with varying degrees of belief.

First stage modules has been previously developed and presented [? ]. In this paper, we concentrate on investigating event inference processing at the upper level of the proposed system.

\subsection{Event Inference Procedure}

Knowledge is the main drive behind the proposed event inference approach. Our knowledge base contains frameworks for representing uncertain events, spatio-temporal relations and event network models, which facilitate atomic event detection, event association and composite event 


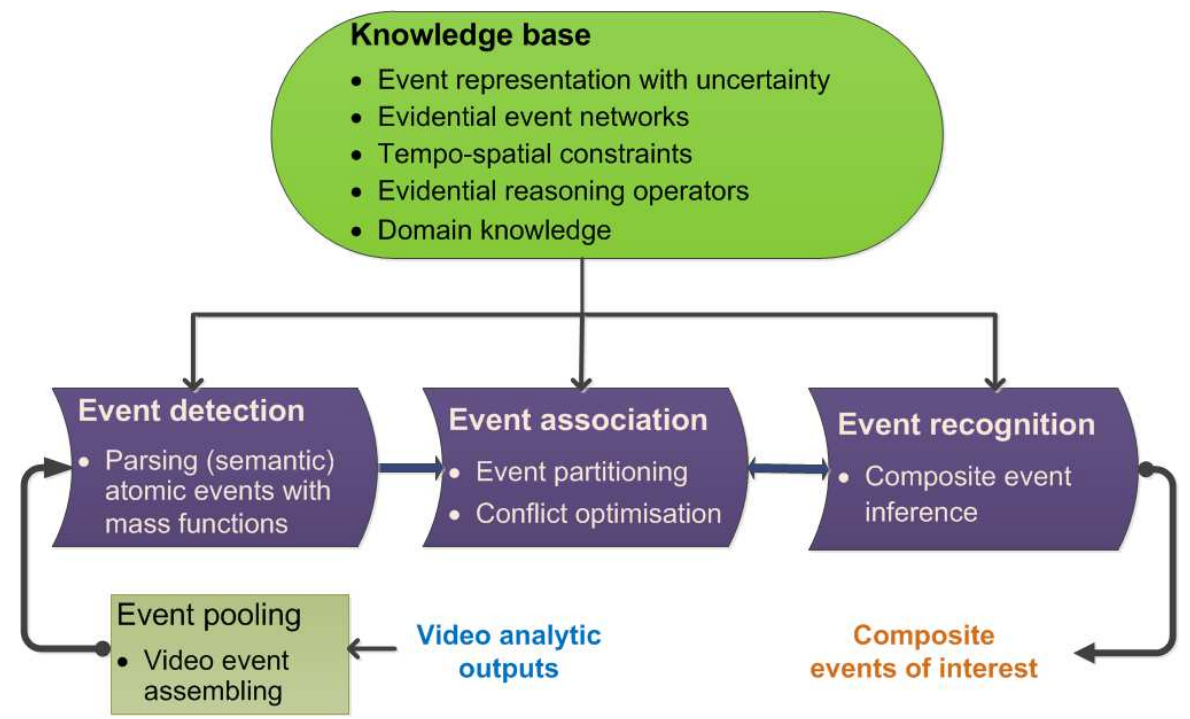

Figure 2: Event inference components

recognition, Fig. 2. Event inference starts by deriving atomic events from the outputs of the computer vision analysis modules. Once atomic events are detected, the event association aims to make the correct association of atomic events to specific subjects. Composite event recognition then is performed on the detected atomic events associated to a single subject. The final outputs of the process are the subjects with the composite events they have undertaken. In the following subsections we will describe the proposed methods for the event inference processing.

\subsection{Event Representation}

Uncertainty is intrinsic to event recognition. Video sensors cannot provide complete information of an evolving scenario over time. In other words, the video analysis modules have certain limitations with respect to providing correct visual information about a scene. During information processing, there is uncertainty in representing the relations between two events of interest. Nevertheless, an intelligent event management system should be able to represent and infer useful information in the presence of uncertainty.

We first define a formal representation of atomic events.

Definition 3. In our event inference system, an atomic event $E$ is represented by a tuple:

$$
E=(\text { eType }, o I D, \text { date, time, location, source, reliaR, } v \text { Frame }, m)
$$

where eType is the descriptor of an event, e.g. "Female Boards the bus"; oID is the identity 
number, assigned by a video analytics module or sensor, for the detected event, e.g. "track id 12"; date is the date of the observed event; time is the time-stamp for the observed event; location presents location information, e.g. "at seat 3" and "a trajectory"; source denotes the source from which the event was detected; reliaR is the degree of reliability of the source; vFrame is the frame of discernment that holds all its values; and $m$ is a mass function on vFrame.

As previously mentioned, in a multi-subject environment, each event, be it atomic or composite, belongs to only one subject. Therefore, to provide a generic framework for the multi-subject scenario that encompasses both atomic and composite events, we introduce the concept of an event node which is defined as follows:

Definition 4. An event node $n$ is a tuple:

$$
n=(\text { eType }, p I D, \text { level }, \text { oID }, \text { date, time, location, source, reliaR, } v \text { Frame }, m)
$$

where eType, oID, date, time, location, source, reliaR, vFrame and $m$ have the same meaning as those in an atomic event; pID represents the identity number of the subject who is responsible for the occurrence of the event; level indicates whether the event is Atomic or Composite.

From the above definition, it can be seen that there are two sorts of event nodes, distinguished by level, either be atomic or composite. For the first type, an event node is an atomic event, except that the event node has an additional element $p I D$. For the second type, an event node represents an event deduced from atomic events and/or composite events. $p I D$ is kept for the same subject through the full sequence and associated to all the event nodes that the subject generates. Therefore, a composite event node has the same $p I D$ as the atomic/composite events that it consists of. Its date and time cover the period from the first event starts until the last event ends. For a composite event node, oID, location, source and reliaR are omitted.

\subsection{Composite Event Modelling}

To represent the hierarchical structure of the relationships between composite and atomic events, and the video analytic outputs, we propose an evidential network model for event composition [? ? ]. 
Definition 5. An evidential event network (EEN) is a graph of an upside-down tree EEN = $(N D, E G, M M)$, where:

- $\boldsymbol{N D}=\left\{n_{1}, \ldots, n_{N}\right\}$ is a set of event nodes;

- $\boldsymbol{E} \boldsymbol{G}$ is a set of directional lines over ND, each of which represents the connection between the nodes at two consecutive layers;

- MM is a set of multi-valued mappings $\Gamma$, each of which describes compatibility relations between the node at the layer where a line starts and the node at the layer where the connection line ends.

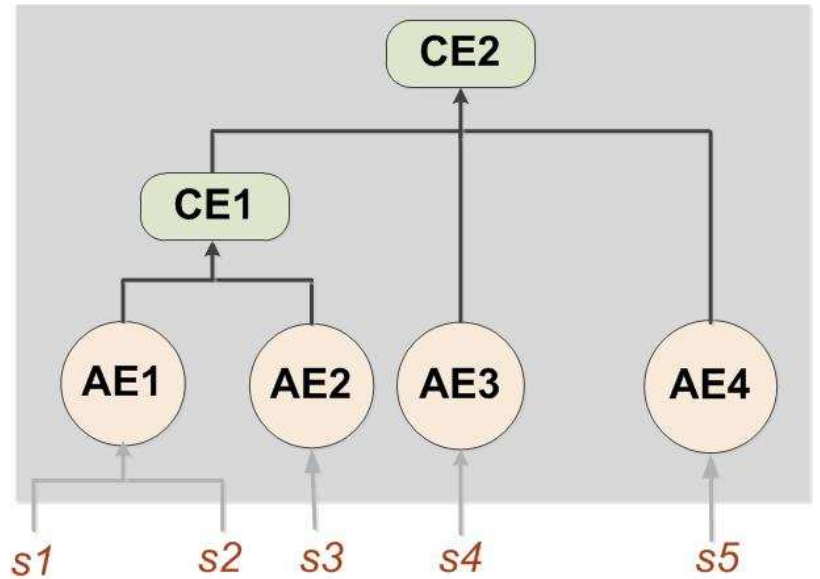

Figure 3: A simple example of the general layout of evidential event networks: s1 - s5 represent sources that provide evidence on atomic events; AE1 - AE4 represent event nodes at atomic level; CE1 and CE2 are the event nodes at composite level.

Fig. 3 shows the layout of an example $E E N, E E N=(N D, E G, M M)$ where

$$
\begin{aligned}
& N D=\{A E 1, A E 2, A E 3, A E 4, C E 1, C E 2\}, \\
& E G=\{A E 1 \longrightarrow C E 1, A E 2 \longrightarrow C E 1, A E 3 \longrightarrow C E 2, A E 4 \longrightarrow C E 2, C E 1 \rightarrow C E 2\}, \\
& M M=\{\Gamma: A E 1 \rightarrow C E 1, \Gamma: A E 2 \rightarrow C E 1, \Gamma: A E 3 \rightarrow C E 2, \Gamma: A E 4 \rightarrow C E 2, \Gamma: C E 1 \rightarrow C E 2\} .
\end{aligned}
$$

On an EEN the nodes are categorised into three levels. The top level contains a root node, and at the bottom level we have many leaf nodes. Between these two levels, the middle level consists 
of several sub-layers. Over the three levels, there exist two types of nodes that are characterised by the level at which a node sits. A leaf node at the bottom level can be an atomic event, such as $A E 1$ in Fig. 3, which is detected by a sensor, e.g. a seat pressure sensor, or a video analytics module, e.g. face detection and a tracker. A leaf node is always connected to the start of an edge. At the other end of the edge, we have nodes from the middle level, such as $C E 1$ in Fig. 3. Middle level nodes are composite events, derived from the connected atomic event nodes. Composite event nodes at this sub-level may be further connected together in order to form composite events at higher sub-layers. On the topmost level of the EEN tree, there is a composite event node that is formed by atomic and/or composite event nodes below, containing the events of interest to the end users.

The hierarchical structure of an EEN reveals semantic relations between events, which are the foundation of evidential event composition and inference developed below. This paradigm also helps in preventing redundancy by reusing the recognised atomic and composite events across EENs.

Uncertainty associated with each node is defined as a mass function $m$. For an atomic event, denoted as a leaf node of the $E E N$, the mass value can be estimated from the accuracy of the computer vision detection module which is its source. For a composite event, the mass distribution can be derived through a composite event inference process as detailed in the following sub-section.

\subsection{Composite Event Inference}

At the bottom level of an EEN, the atomic events as leaf nodes are detected from outputs of sensors or video analysis modules. Information on detected atomic event nodes can be used to deduce information on higher-level nodes of composite events by propagating and aggregating evidence of atomic events through the network using evidential reasoning operations.

Composite event inference starts from having detected atomic events from outputs of the computer vision analysis modules and moving up within an EEN. The final output of the process is the mass function on the composite event node in concern. Algorithm 1 details the inference process. 


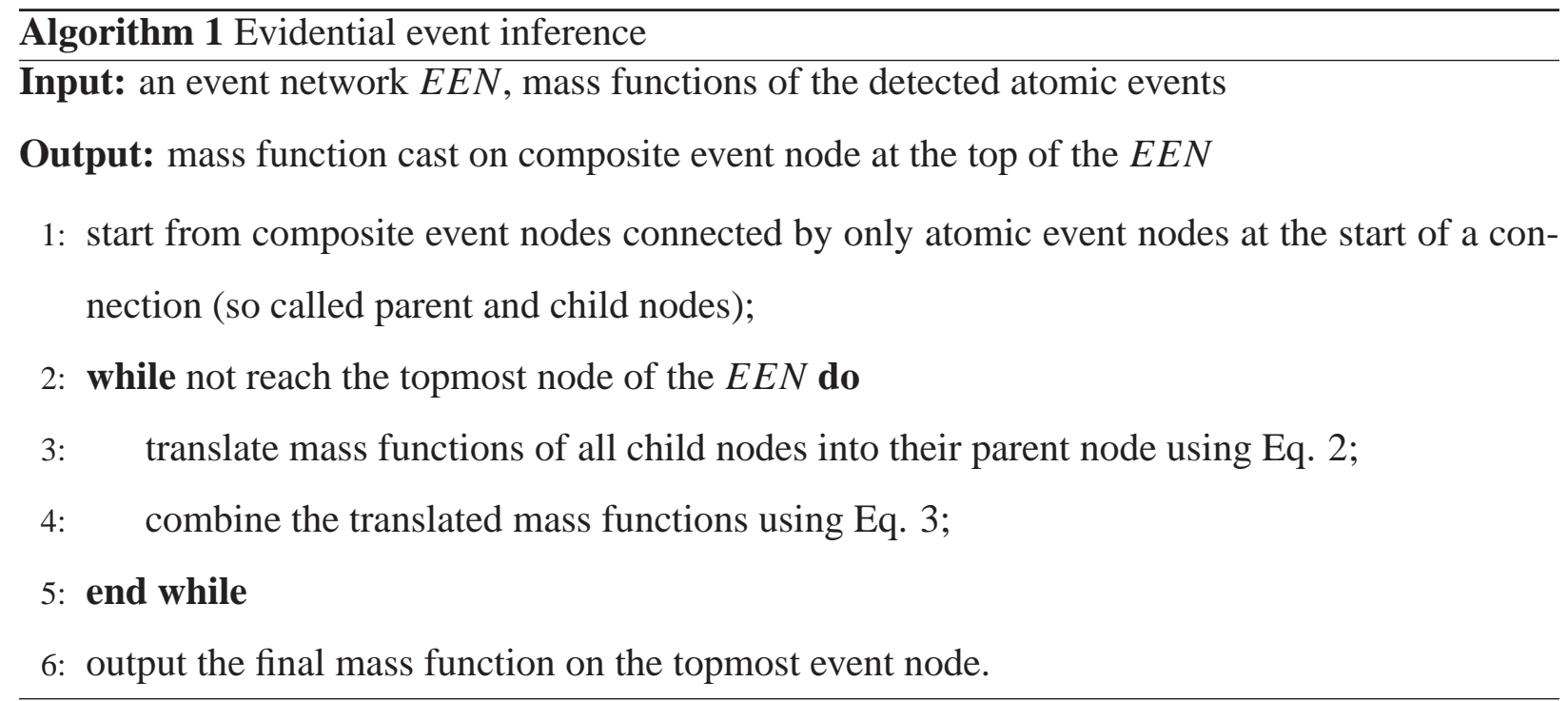

\subsection{Event-Subject Association}

In multi-subject scenarios, it is usual that several subjects may be present at the same time, resulting in highly ambiguous video analytic output. For example, it is quite common that a single individual is assigned several IDs in complex scenes due to split/erroneous tracks produced by the tracking system. Intuitively arranging all detected atomic events with the same object ID assigned by video analytics into a composite event network $E E N$ and directly making inference on the composite event node at EEN's root inevitably produces errors. To solve this problem, we propose an atomic event association method by integrating the use of temporal relation modelling in event composition and evidential reasoning in event inference.

The event association problem can be seen as the association of all related atomic events with an individual under observation. The problem is two-fold: (i) partitioning a set of atomic events into different groups, and (ii) selecting the most probably set of partitions among many possible sets.

Definition 6. For a set of atomic events $\Xi=\left\{E_{1}, \ldots, E_{|\Xi|}\right\}$, a partitioning $S=\left\{S_{1}, \ldots, S_{|S|}\right\}$, satisfies:
(1) $S_{1} \cup \cdots \cup S_{|S|}=\Xi$
(2) $S_{i} \neq \emptyset$
(3) $S_{i} \cap S_{j}=\emptyset$

where $i, j=1, \ldots,|S|$ and $i \neq j$. 
It is possible that we do not have sufficient information to justify if an atomic event belongs to one subject or another. This results in many possible choices to group those atomic events, i.e. we have many ways for partitioning. In the cases where several possible sets of partitions exist, a partition set may be considered more satisfying than others and is therefore selected as the most optimum partitioning of the atomic events.

\subsubsection{Event Partitioning}

Partitioning atomic events aims to identify subjects who are responsible for the occurrences of the atomic events, in order to infer the composite events undertaken by the subjects. We investigate the intrinsic properties of ID assignments, as well as characteristics of atomic events, in order to determine a possible partitioning. For this purpose, we introduce two functions $\Phi$ and $\Psi$.

Let $P I D=\left\{p I D_{1}, \ldots, p I D_{P}\right\}$ be a set of subject IDs, $\Xi=\left\{E_{1}, \ldots, E_{|\Xi|}\right\}(|\Xi| \geq P)$ be a set of atomic events, and $S=\left\{S_{1}, \ldots, S_{|S|}\right\}$ be a partitioning of $\Xi$. For $\Xi$, we have $\Omega=$ $\left\{e_{1}, \neg e_{1}, \ldots, e_{|\Xi|}, \neg e_{|\Xi|}\right\}$, a set of possible states for all the atomic events related to a subject, whereas $e_{i}$ means the occurrence of event $E_{i}$ concerns the subject, and $\neg e_{i}$ does not.

Definition 7. A function $\Phi$ that assigns a partition $S_{i}$ to a subject ID $p I D_{i}$ is defined as:

$$
\Phi\left(p I D_{i}\right)=S_{i}
$$

where $S_{i} \subseteq S, i=1, \ldots,|S|$.

A mapping function $\Phi$ represents the one-to-one mapping relation between a subject ID and a partition of atomic events.

Definition 8. A function $\Psi$ that maps each subject ID pID $D_{i}$ onto possible states of the atomic events is defined as follows:

$$
\Psi\left(p I D_{i}\right)=\omega_{i}
$$

where $\omega_{i} \subset \Omega$ and $\nexists E_{j} \in \Xi$, s.t. $\left\{e_{j}, \neg e_{j}\right\} \subseteq \omega_{i}$.

A mapping function $\Psi$ represents the relation between a subject ID and the occurrence/nonoccurrence states of atomic events. 
There is a relation between an event $E_{j}$ and the sate set $\left\{e_{j}, \neg e_{j}\right\}$ based on the two mapping function $\Phi$ and $\Psi$.

$$
\left\{\begin{array}{ll}
E_{j} \in S_{i} & \Leftrightarrow e_{j} \in \omega_{i} \\
E_{j} \notin S_{i} & \Leftrightarrow \neg e_{j} \in \omega_{i}
\end{array} \quad \text { for any } i=1, \ldots, P, j=1, \ldots, m .\right.
$$

From Definition 7 and 8, we can see that event partitioning is actually about deciding the state of each atomic event in relation to a subject ID. For a subject $p I D_{i}$, we can have a set of states of the detected atomic events, where the occurrence/non-occurrence state of an atomic event indicates that $p I D_{i}$ is responsible for the happening of the atomic event. The restrictions of $\omega_{i} \subset \Omega$ and $\left\{e_{j}, \neg e_{j}\right\} \nsubseteq \omega_{i}$ for any $j \in[1,|\Xi|]$ means that, for a given subject ID, either the occurrence or non-occurrence state of an atomic event holds.

To deduce the possible state of an atomic event for a subject, we consider the occurrence constraints on atomic events concerning the subject. For a subject, the occurrence of an atomic event can be affected by and/or has impacts on the occurrence of other atomic events. For example, "I am reading a book at home at 9 pm" implies that "I cannot be playing basketball at a sports centre at $9 \mathrm{pm}$ on the same day". Identifying the state of an atomic event from the already known states of another atomic events is called event implication. This is managed by using constraint rules, which determine the possible state of an atomic event with regard to other atomic events in concern.

Definition 9. A constraint rule $R$ is expressed as a tuple

$$
R=(\text { S tatement }, \text { Premise }, \text { Condition }, \text { Result })
$$

where:

Statement is the description of the constraint rule that the premise set should obey.

Premise is a set of eTypes of which atomic events are prerequisites.

Condition is a conjunction of a set of conditions on the states of some atomic events currently hold. 
Result is a set of the states of atomic events in relation to a subject, obtained by applying the constraint rule.

In this work, we consider three types of constraints: temporal, spatial and common knowledge. Since atomic events happen over a period of time, the temporal relation between atomic events usually imply their states in relation to each other. For example, a man cannot play basketball and watch TV at the same time. Similar to temporal constraints, spatial relations between atomic events usually implies their states. For example, a man cannot be in two separate places at the same time. A common knowledge constraint is derived from knowledge about the domain context. Consider a man taking a bus, he cannot exit the bus without boarding the bus first. A constraint rule can include one, two or all three types of constraints.

Condition in the form of formula presents temporal and spatial relations of existing atomic events. In particular Allen's temporal relation models are used to describe temporal relations between two event instances. We abstract the Allen's relations in Table 1, into a small set, $\{b, a, m, m i, o l, e q\}$, as shown in Table 2.

Table 2: Mapping of the abstract and original Allen's interval temporal relations

\begin{tabular}{|c|c|}
\hline Abstract relation & Allen's relation(s) \\
\hline$b$ & $b$ \\
\hline$a$ & $m$ \\
\hline$m$ & $m i$ \\
\hline$m i$ & $o, o i, s, s i, f, f i, d, d i$ \\
\hline$o l$ & $e q$ \\
\hline$e q$ & \\
\hline
\end{tabular}

Rules are pre-requisite for finding states of atomic events. Rule $R$ is used to search for events that violate or obey constraints of the three types. Therefore, the state of an atomic event can be identified in relation to a subject. Upon the states of all atomic events have been determined for each subject, the partitions of atomic events can then be obtained.

To show what an event constraint rule looks like, consider two examples from the bus journey 
scenario. Assume that the atomic events are derived from the video data. From common sense existentialism, we can have the following rules.

Example 1. A rule, ensuring that one person cannot undertake two different events at a time that are detected in a bus scenario, can be defined as follows:

\section{Rule RI}

Statement: a person can not undertake two events at a time;

Premise: $\{P B, P M, \text { PSIT, PSTD, PE }\}^{1}$ is a set of event types;

Condition: $E_{i}$.Time eq $E_{j}$.Time $\wedge e_{i} \in \Psi\left(p I D_{p}\right)$;

Result: $\neg e_{j} \in \Psi\left(p I D_{p}\right)$.

Example 2. A rule, describing that a person cannot exit a bus before having boarded the bus, can be defined as follows:

\section{Rule R2}

Statement: a person can only exit the bus after having boarded the bus;

Premise $=\{P B, P E\}$ is a set of event types;

Condition: $E_{i}$. eType $=P B \wedge E_{j}$.eType $=P E \wedge E_{i}$.Time a $E_{j}$.Time $\wedge e_{i} \in \Psi\left(p I D_{p}\right)$;

Result: $\neg e_{j} \in \Psi\left(p I D_{p}\right)$.

Based on the relevance of the events detected by the video analytics and the non-relevance of the events obtained by the implication rule, we attempt to find the optimum partitioning for the set of atomic events. A partitioning of the set of atomic events is to identify the persons under observation, each partition $S_{i} \subset S$ should satisfy the following principle:

Proposition 1. Suppose PID $=\left\{p I D_{1}, \ldots, p I D_{P}\right\}$ is a set of possible person IDs for a set of atomic events $\Xi=\left\{E_{1}, \ldots, E_{|\Xi|}\right\}(|\Xi|>P), \Omega=\left\{e_{1}, \neg e_{1}, \ldots, e_{|\Xi|}, \neg e_{|\Xi|}\right\}$ is a set of possible states for all atomic events in $\Xi$, and $\Psi$ is a mapping function that indicates the relation between subject ID and the states of the given atomic events, then we have:

(i) Uniqueness: $\quad \exists e_{i} \in \Omega$, s.t. $\left.e_{i} \in \Psi\left(p I D_{u}\right) \cap \Psi\left(p I D_{v}\right)\right), u, v=1, \ldots, P, u \neq v$;

\footnotetext{
${ }^{1} P B$ - person boarding, $P M$ - person moving, $P S I T$ - person sitting, $P S T D$ - person standing, $P E$ - person exiting.
} 
(ii) Completeness: $\quad \Psi\left(p I D_{1}\right) \cup \ldots \cup \Psi\left(p I D_{P}\right)=\Omega$.

Proof. (i) Uniqueness: Assume that $\exists e_{k} \in \Omega$, s.t. $e_{k} \in \Psi\left(p I D_{u}\right) \cap \Psi\left(p I D_{v}\right), u, v=1, \ldots P, u \neq v$, by Definition 8 , we have $E_{k} \in S_{u}$ and $E_{k} \in S_{v}$. Thus, $S_{u} \cup S_{v} \neq \emptyset$. It violates the definition of Partition in Definition 6. Thus, item (i) holds.

(ii) Completeness: We first prove that $\left(\Psi\left(p I D_{1}\right) \cup \cdots \cup \Psi\left(p I D_{P}\right)\right) \subset \Omega$.

By Definition 8 , we have $\Psi\left(p I D_{i}\right) \subset \Omega(i=1, \ldots, P)$. Thus, it holds.

Then, we prove that $\Omega \subset\left(\Psi\left(p I D_{1}\right) \cup \cdots \cup \Psi\left(p I D_{P}\right)\right)$.

Let $S_{1} \cup \cdots \cup S_{P}$ be a possible partitioning of $\Xi$ that indicates the set of possible person IDs PID. By Definition 6, for any $E_{i} \in \Xi$, we have $E_{i} \in\left(S_{1} \cup \cdots \cup S_{|S|}\right)$. Moreover, for any state $x, x \in \Omega$, we have $\exists E_{k} \in \Xi$, such that, $x \in\left\{e_{k}, \neg e_{k}\right\}$, where $E_{k} \in\left(S_{1} \cup \cdots \cup S_{|S|}\right)$. Without losing generality, let $E_{k} \in S_{l}, S_{l} \in\left\{S_{1}, \ldots, S_{P}\right\}$. Then, by Definition 6, for any $S_{h}(h \neq l)$, we have $S_{h} \cup S_{l}=\emptyset$ and $E_{k} \notin S_{h}$. Thus, by Definition 8, we have $e_{k} \in \Psi\left(p I D_{l}\right)$ and $\neg e_{k} \in \Psi\left(p I D_{h}\right)$. Clearly, we have $x \in \Psi\left(p I D_{l}\right) \cup \Psi\left(p I D_{h}\right)$ for any $h \neq l$. So, for any occurrence state $x$, if $x \in \Omega$, then $x \in\left(\Psi\left(p I D_{1}\right) \cup \cdots \cup \Psi\left(p I D_{P}\right)\right)$. Thus, item (ii) holds.

The completeness states that any atomic event shall be included in a partition. The uniqueness means that an atomic event should be in one and only one partition.

Following Proposition 1, we obtain all the possible partitions for a set of atomic events, indicating the occurrence/non-occurrence states of atomic events that each subject ID holds. The next step is to determine which partition minimises the inferred conflict.

\subsubsection{Minimum Conflict Optimisation}

After obtaining a possible set of partitions for all atomic events, we can assign each partition to a possible person ID, i.e. we have an one-to-one mapping from $S=\left\{S_{1}^{t}, \ldots, S_{P}^{t}\right\}$ to $P I D^{t}=$ $\left\{p I D_{1}^{t}, \ldots, p I D_{P}^{t}\right\}^{2}$. Therefore, we can obtain a set of event nodes for each possible person ID defined in Definition 4. Afterwards, we apply EENs introduced in 4.4 to infer all the composite

\footnotetext{
${ }^{2}$ Since there may be more than one possible partitioning for a set of atomic events, we use the superscript $t$ to distinguish them.
} 
events related to each possible person ID. However, if we have more than one possible partitioning of the atomic events, how can we choose the best from many possibilities? In this subsection, we will solve this problem using the conflict factor in the Dempster's rule of combination.

After having identified all the atomic events related to a specific subject, we feed the atomic events into the EENs and derive the composite events. This is done by aggregating atomic events through EENs using the Dempster's Rule of Combination as proposed in the section 4.5. When combining atomic event evidence, the conflict factor $k$ in Eq. 3 is a measure of the amount of conflict between the two pieces of evidence as described below.
(1) $k=0$
totally agree;
(2) $0<k<1$
agree to some extent;
(3) $k=1$
totally disagree.

We use $k$ to select the most probable partition of object IDs. Since each composite event for a possible person ID accompanies a degree of conflict, we need to consider the aggregation effect during the inference process for each possible partitioning.

Definition 10. Let $S_{1}^{t} \cup \cdots \cup S_{P}^{t}$ be a possible partitioning of the set of atomic events $\Xi=$ $\left\{E_{1}, \ldots, E_{|\Xi|}\right\}$ and $P$ be the total number of persons, where for each $S_{p}^{t}$, we have $S_{p}^{t}=\left\{E_{u}, \ldots, E_{v}\right\}$, each element of which relates to the pth person. We therefore calculate the aggregation effect in terms of a conflict factor when inferring composite events for each possible person, denoted as $\hat{k}_{p}^{t}$ :

$$
\hat{k}_{p}^{t}=\frac{\sum_{i=1}^{L} k_{i}^{t}}{L}
$$

where $L$ is the total number of the composite events inferred for the pth person. $k_{i}^{t}$ is a conflict factor obtained from the inference of the ith composite event, as $k$ in Eq. 3.

For a conflict factor, the smaller its value is, the more confident support evidence has. From this we can have the definition the most probable partitioning.

Definition 11. The dth possible partitioning is the most probable one for the set of object IDs if it satisfies $d=\arg \min _{t}\left(k^{t}\right), k^{t}=\sum_{p=1}^{P} \hat{k}_{p}^{t}$. 
After finding the most possible partition for the set of subject IDs, we retain a set of person IDs based on Definition 7 and the event nodes that have been determined to them by using Definition 4.

Algorithm 2 summarises the event association process.

\section{Experiments}

In this section we describe an experiment in which the ability of our system to recognise the following four composite events is measured:

- MBTS: Male boards, moves to a seat and sits down

- FBTS: Female boards, moves to a seat and sits down

- PCS: Person changes seat

- PEX: Person exits

We compare the performance of our system to a simple rules-based approach with no reasoning and an adapted Bayesian reasoning system.

\subsection{Environmental Set-Up}

We hired a standard single-deck bus from Translink (Northern Ireland), which travelled a defined journey in the Northern Ireland Science Park. Fig. 4a is the aerial view of the local neighbourhood with a red curve outlining the route and six black circles marking six bus stops. The researchers from the ECIT centre were recruited as passengers. The bus saloon and the seat plan are shown in Fig. $4 b$ and $4 c$, respectively. In the experiments twenty seats in the first five rows of the bus, numbered $\mathrm{C} 1-\mathrm{C} 20$, were deployed as passenger seats.

Two cameras were used on the bus: a Panasonic camera WV-NP244 (camera A) is used to monitor the front door of the bus, and an AXIS M31-R camera (camera B) is used to monitor the saloon area. Camera A is carefully positioned so that it can capture a passenger's face as s/he boards the bus. Camera B looking at the saloon can record the movements of passengers. 


\section{Algorithm 2 Event association}

Input: $\Xi=\left\{E_{1}, \ldots, E_{|\Xi|}\right\}$, a set of atomic events;

$P$ the number of persons;

EEN the evidential event networks;

$R$ constraint rules;

Output: $S=\left\{S_{1}, \ldots, S_{P}\right\}$, a set of atomic event partitions

\section{Begin}

1: $\Omega=\left\{e_{1}, \neg e_{1}, \ldots, e_{|\Xi|}, \neg e_{|\Xi|}\right\}$;

2: initialise $\omega_{1}=\cdots=\omega_{P}=\Omega$;

3: $i=1$;

4: while not reach the end of $\Xi$ do

5: $\quad$ Search $\omega_{1}, \ldots, \omega_{P}$ to find all $\omega_{j}$ that hold events satisfying the constraints on $E_{i}$;

6: if possible then

7: $\quad$ Delete $e_{i}$ or $\neg e_{i}$ accordingly from $\omega_{j}$;

8: $\quad$ else

9: $\quad$ Create the options;

10: $\quad$ end if

11: $\quad i++$

12: end while

13: Find all the combinations of elements in $\omega_{j}^{t}$ by proposition 1 ;

14: Calculate $k$ for each combination;

15: Select $\omega^{t}$ holding the smallest $k$ as the association;

16: Obtain the partitioning $S^{t}$ from $\omega^{t}$;

17: Output the partitioning $S^{t}$.

\section{End}



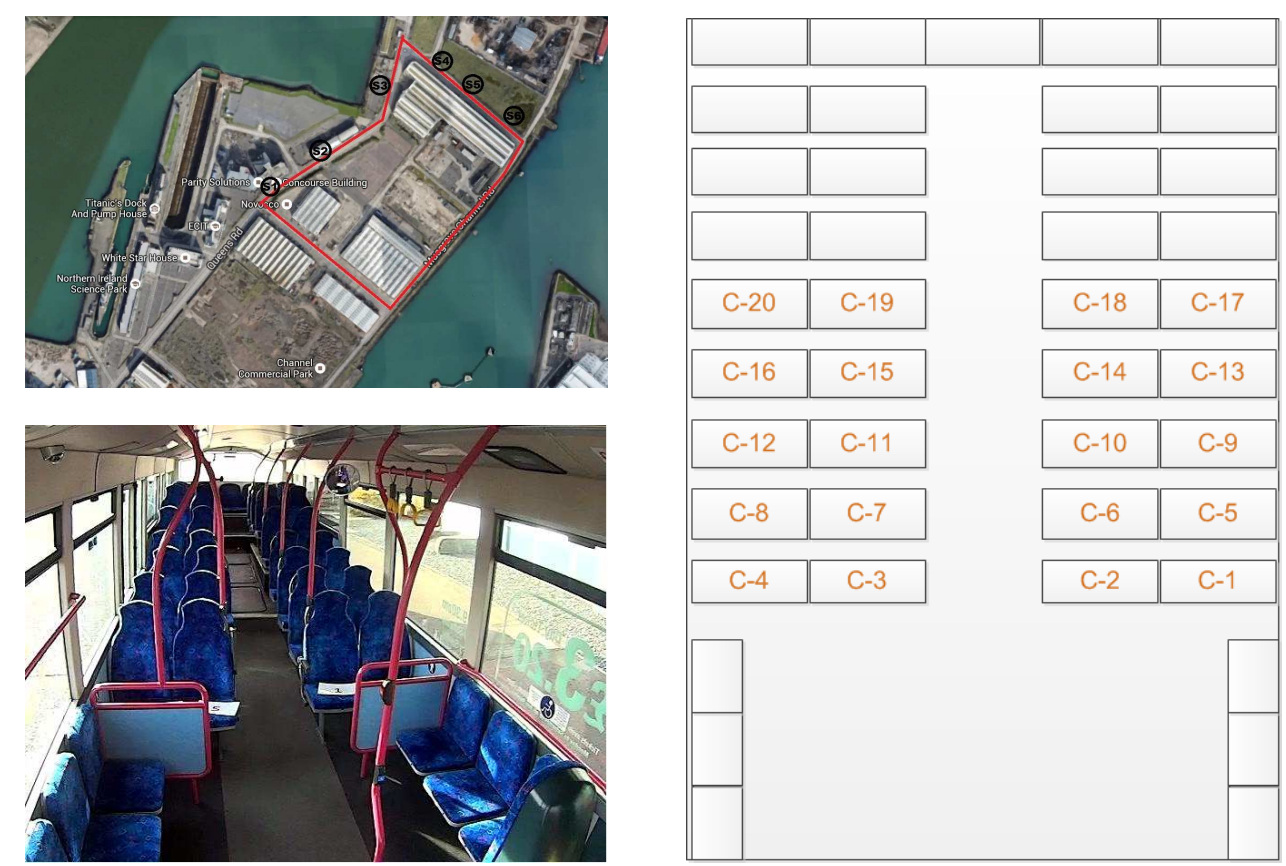

Figure 4: Experimental Environment: (a) route with six designated stops (the red curve highlights the route, the black circles mark the six bus stops) (b) bus saloon (c) seat layout (numbered seats are used in experiments)

\subsection{Dataset}

We captured eight sequences of varying complexity, including different numbers of passengers on board, various passenger behaviour patterns, and from simple to difficult scene captures. The properties of the eight sequences are summarised in Table 3. Each sequence is described in detail in Appendix C.

Table 3: Properties of the eight test sequences

\begin{tabular}{|c|c|c||c|c|c|}
\hline Sequence & No. of passengers & No. of Frames & Sequence & No. of passengers & No. of Frames \\
\hline 1 & 1 male and 1 female & 2556 & 5 & 1 male and 1 female & 1902 \\
\hline 2 & 1 male and 1 female & 1733 & 6 & 2 male and 1 female & 5202 \\
\hline 3 & 1 male and 1 female & 2667 & 7 & 2 male and 2 female & 5522 \\
\hline 4 & 1 male and 1 female & 2662 & 8 & 3 male and 3 female & 10322 \\
\hline
\end{tabular}




\subsection{Video Processes}

For detecting passengers boarding and gender recognition, we employ a camera pointing at the door of the bus. The well-known Jones and Viola face detector is then applied to the acquired video. The output of this detection is then input to a face-based gender classifier. This, firstly, projects the face image onto a subspace derived using a principal component analysis of a training data set of face images. The resulting feature is input to a support vector machine that has been trained on approximately two thousand male and female face images. The resulting output is the credibility of the face as being either female or male (Fig. 5a and 5b).

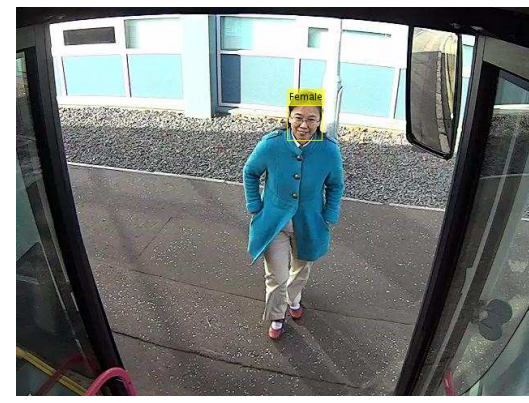

(a)

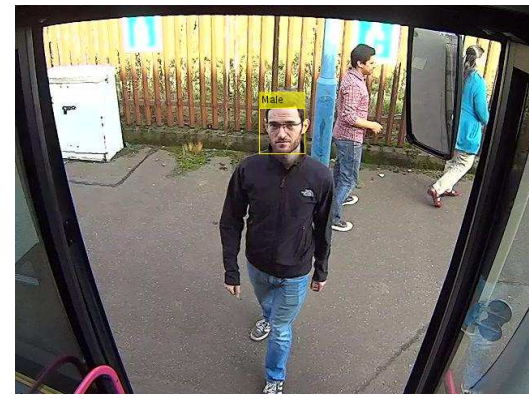

(b)

Figure 5: Gender classification

For monitoring movements of passengers, we employ a 3D tracker that consists of three stages. Firstly, we apply the Poselet detector to detect instances of humans in the video on a frame-byframe basis. These detections are then linked together to form tracks using a hierarchical linear assignment procedure. In the first level, detections are linked on a frame-to-frame basis by linear assignment. The resulting tracklets are then subsequently linked into tracks by a second level of linear assignment (see [? ] for further details). Fig. 6a shows an example of a male and a female being tracked and their corresponding tracks projected into real-world space, Fig.6b.

For sitting and standing detection, the height from the top of the head of an individual to the ground plane is calculated and compared to a threshold of $1.4 \mathrm{~m}$. This threshold was empirically determined through trial and error, and is around the lower end of the normal human height distribution. The height can be estimated given the scene calibration and a standard reference height in the scene. When a passenger boards the bus, the passenger is inferred to be standing. For sub- 


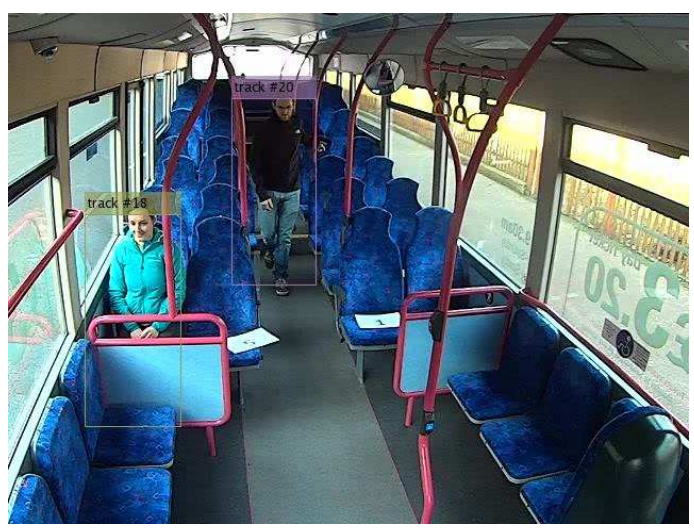

(a)

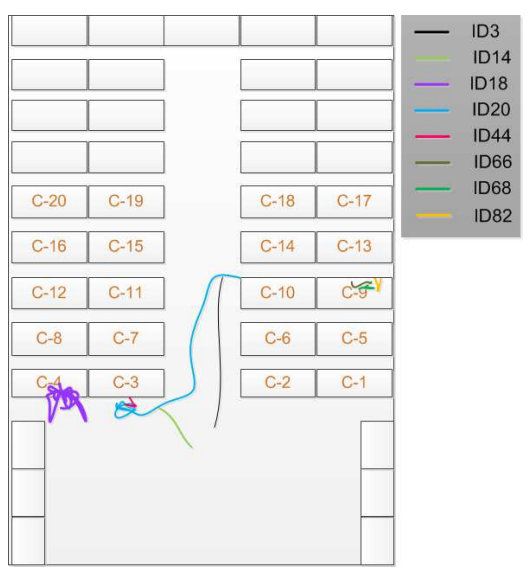

(b)

Figure 6: Outputs of the tracker: (a) image with tracker bounding boxes over the person in the scene (b) the corresponding track plots in real-world coordinates

sequent frames, if the height falls below the threshold at any point, we infer that the passenger has sat down. Whilst sitting, if the height increases beyond the threshold value, we infer that the passenger has just stood up. Fig. 7 shows an example of sitting and standing being detected.

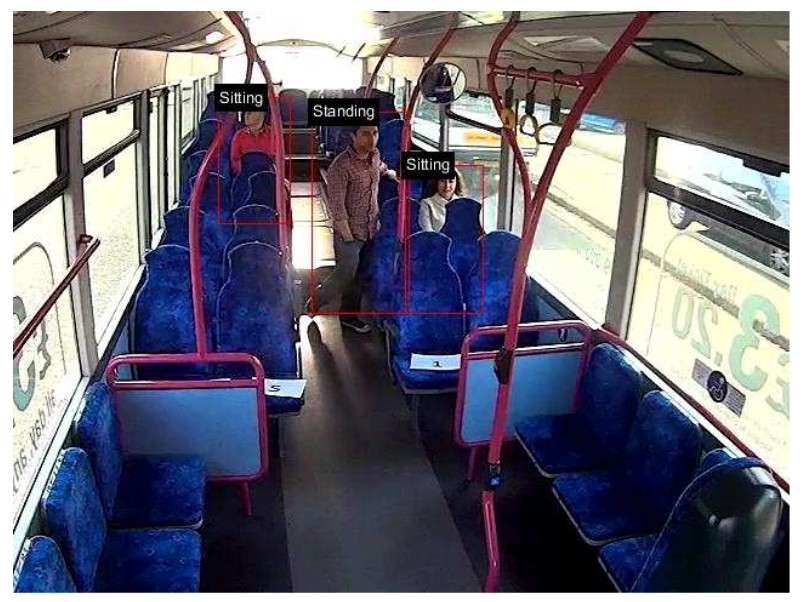

Figure 7: Sitting and Standing detection

\subsection{Evaluation}

To evaluate the performance of our system in terms of the association of events with personal IDs and composite event recognition, we use two measurements. The first of these is the accuracy 
of the event association, $A$, which is given by

$$
A=\frac{C_{A E}}{N_{A E}}
$$

where $C_{A E}$ is the number of atomic events in a sequence correctly associated with a personal ID, and $N_{A E}$ is the total number of atomic events in the sequence. The second is the accuracy of the composite event recognition, $R$, given by

$$
R=\frac{1}{N_{C E}} \sum_{i=1}^{N_{C E}} I_{i}
$$

where $I_{i}$ equals one if the $i^{\text {th }}$ recognised composite event in the sequence matches the ground truth, and zero if not, and $N_{C E}$ is the total number of composite events in the sequence.

For the purposes of this each sequence is manually ground truthed both in terms of its atomic

\begin{tabular}{|c|c|c|c|c|c|c|c|c|c|c|}
\hline \multicolumn{11}{|c|}{ Groundtruth - seq5: part 1} \\
\hline & \multirow[b]{2}{*}{ Gender } & \multicolumn{2}{|c|}{ Atomic event 1} & \multicolumn{4}{|c|}{ Atomic event 2} & \multicolumn{3}{|c|}{ Atomic event 3} \\
\hline & & Title & End frame & IDs & Title & End_fr & frame & IDs & Title & End_frame \\
\hline \multirow{2}{*}{ P1 } & \multirow{2}{*}{ Female } & FB & 88 & 7 & PM & 265 & & Seat9 & Sit_dow & n 315 \\
\hline & & \multicolumn{9}{|c|}{ Composite Event 1: FBTS9 } \\
\hline \multirow{2}{*}{ P2 } & \multirow{2}{*}{ Male } & MB & 821 & 70 & PM & 979 & & Seat 13 & Sit_dow & 1019 \\
\hline & & \multicolumn{9}{|c|}{ Composite Event 1: MBTS13 } \\
\hline \multicolumn{11}{|c|}{ Groundtruth - seq5: part 2} \\
\hline \multicolumn{3}{|c|}{ Atomic event 4} & \multicolumn{4}{|c|}{ Atomic event 5} & \multicolumn{4}{|c|}{ Atomic event 6} \\
\hline IDs & Title & End_frame II & IDs & \begin{tabular}{l|l} 
Title \\
\end{tabular} & \begin{tabular}{l|l} 
End \\
\end{tabular} & frame & e IDs & & Title & End frame \\
\hline Seat9 & \multicolumn{2}{|c|}{ Stand up 1133} & 84 & PM & \begin{tabular}{l|l} 
& 124 \\
\end{tabular} & & Sea & at3 & Sit_down & 1246 \\
\hline \multicolumn{11}{|c|}{ Composite Event 2: PCS3 } \\
\hline Seat13 & \multicolumn{2}{|c|}{\begin{tabular}{|l|l|} 
Stand_up 1250 \\
\end{tabular}} & $105,133,13$ & \begin{tabular}{|l|l|}
66 & $\mathrm{PM}$ \\
\end{tabular} & \begin{tabular}{l|l|} 
& 144 \\
\end{tabular} & & $\mathrm{Sez}$ & at4 & Sit_down & 1443 \\
\hline \multicolumn{11}{|c|}{ Composite Event 2: PCS13 } \\
\hline \multicolumn{11}{|c|}{ Groundtruth - seq5: part 3} \\
\hline \multicolumn{3}{|c|}{ Atomic event 7} & \multicolumn{4}{|c|}{ Atomic event 8} & \multicolumn{4}{|c|}{ Atomic event 9} \\
\hline IDs & Title & End_frame & IDs & Title & End_f & rame II & IDs & & itle $\quad$ E & End frame \\
\hline Seat3 & \multicolumn{2}{|c|}{ Stand up 1638} & 162 & PM & 1700 & & -15 & & $E$ & 1765 \\
\hline \multicolumn{11}{|c|}{ Composite Event 3: PEX } \\
\hline Seat4 & \multicolumn{2}{|c|}{ Stand_up 1725} & 199 & PM & 1798 & & -12 & $\mathrm{P}$ & $E$ & 1875 \\
\hline \multicolumn{11}{|c|}{ Composite Event 3: PEX } \\
\hline
\end{tabular}
events and its composite events. Fig. 8 shows the manual ground truth for sequence 5. From

Figure 8: Manual ground truth for sequence 5.

the table we can see that both male and female have nine atomic events each consisting of the 
sequence: PB, PM, PSIT, PSTD, PM, PSIT, PSTD, PM and PE (PB = "male or female boards bus", $P M=$ "person moves from X to Y", PSIT = "person sits", PSTD = "person stands" and $P E=$ "person exits"). Similarly, these correspond to three composite events: MBTS(FBTS), PCS and PEX. Each sequence was then input to our system and the corresponding atomic events, their associated person IDs and the recognised composite events output were recorded. Comparison of these against the ground truth enabled us to calculate both $A$ and $R$ for each sequence.

\subsection{Results and Analysis}

Table 4 shows the variation in $A$ with sequence numbers. Clearly, the event association works very well for almost all the sequences apart from 8 , almost above $90 \%$ on $A$ for each sequence. The last column in Table 5 shows the $R$ values obtained for each sequence with our evidential reasoning system. The event recognition achieves $100 \%$ of $R$ for four sequences, $90 \%$ for one sequence, $83 \%$ for one sequence, and $80 \%$ for one sequence, lower than $50 \%$ for one sequence.

Table 4: Association results for the evidential reasoning system

\begin{tabular}{|c|c|c|c|}
\hline \multirow{2}{*}{ sequence } & \multicolumn{2}{|c|}{ number of atomic events } & \multirow{2}{*}{$\mathrm{A}(\%)$} \\
\hline & Ground Truth & Evidential Reasoning System & \\
\hline 1 & 12 (2 PB, 4 PM, 2 PSIT, 2 PSTD, 2 PE) & 12 (2 PB, 4 PM, 2 PSIT, 2 PSTD, 2 PE) & 100 \\
\hline 2 & 15 (2 PB, 5 PM, 3 PSIT, 3 PSTD, 2 PE) & 13 (2 PB, 5 PM, 2 PSIT, 2 PSTD, 2 PE) & 87 \\
\hline 3 & 15 (2 PB, 5 PM, 3 PSIT, 3 PSTD, 2 PE) & 15 (2 PB, 5 PM, 3 PSIT, 3 PSTD, 2 PE) & 100 \\
\hline 4 & 6 (1 PB, 2 PM, 1 PSIT, 1 PSTD, 1 PE) & 6 (1 PB, 2 PM, 1 PSIT, 1 PSTD, 1 PE) & 100 \\
\hline 5 & 18 (2 PB, 6 PM, 4 PSIT, 4 PSTD, 2 PE) & 16 (2 PB, 6 PM, 3 PSIT, 3 PSTD, 2 PE) & 89 \\
\hline 6 & 21 (3 PB, 7 PM, 4 PSIT, 4 PSTD, 3 PE) & 21 (3 PB, 7 PM, 4 PSIT, 4 PSTD, 3 PE) & 100 \\
\hline 7 & 27 (4 PB, 9 PM, 5 PSIT, 5 PSTD, 4 PE) & 22 (4 PB, 6 PM, 4 PSIT, 4 PSTD, 4 PE) & 81 \\
\hline 8 & 39 (6 PB, 13 PM, 7 PSIT, 7 PSTD, 6 PE) & 24 (6 PB, 8 PM, 5 PSIT, 4 PSTD, 1 PE) & 62 \\
\hline
\end{tabular}

Analysis reveals that for sequences 2, 5 and 7 the $\mathrm{R}$ values were less than $100 \%$. For sequence 2 and 5 there lacked sitting detections and tracking. This resulted in the PM and PSIT atomic events being incorrect and undetected, which in turn resulted in the composite event $\mathrm{P}(\mathrm{M} / \mathrm{F}) \mathrm{BTS}$ being incorrect. Sequence 2 contains five composite events, the resulting $R$ value was $80 \%$. Sequence 5 contains six composite events, the resulting $R$ value was $83 \%$. For sequence 7 there were two 
Table 5: Recognition results for rule-based approach, Bayesian approach, and our evidential reasoning approach

\begin{tabular}{|c|c|c|c|c|}
\hline \multirow{2}{*}{ sequence } & \multicolumn{4}{|c|}{ number of composite events - R } \\
\hline & Ground Truth & Rule-based & Bayesian reasoning & Evidential Reasoning \\
\hline \multirow{2}{*}{1} & 4 & $3-75 \%$ & $4-100 \%$ & $4-100 \%$ \\
\hline & 2 PBTS, 2 PEX & 1 PBTS, 2 PEX & 2 PBTS, 2 PEX & 2 PBTS, 2 PEX \\
\hline \multirow{2}{*}{2} & 5 & $4-80 \%$ & $2-40 \%$ & $4-80 \%$ \\
\hline & 2 PBTS, 1 PCS, 2 PEX & 1 PBTS, 1 PCS, 2 PEX & 1 PBTS, 1 PCS, 0 PEX & 1 PBTS, 1 PCS, 2 PEX \\
\hline \multirow{2}{*}{3} & 5 & $2-40 \%$ & $5-100 \%$ & $5-100 \%$ \\
\hline & 2 PBTS, 1 PCS, 2 PEX & 0 PBTS, 0 PCS, 2 PEX & 2 PBTS, 1 PCS, 2 PEX & 2 PBTS, 1 PCS, 2 PEX \\
\hline \multirow{2}{*}{4} & 2 & $0-0 \%$ & $2-100 \%$ & $2-100 \%$ \\
\hline & 1 PBTS, 1 PEX & 0 PBTS, 0 PEX & 1 PBTS, 1 PEX & 1 PBTS, 1 PEX \\
\hline \multirow{2}{*}{5} & 6 & $5-83 \%$ & $4-67 \%$ & $5-83 \%$ \\
\hline & 2 PBTS, 2 PCS, 2 PEX & 1 PBTS, 2 PCS, 2 PEX & 0 PBTS, 2 PCS, 2 PEX & 1 PBTS, 2 PCS, 2 PEX \\
\hline \multirow{2}{*}{6} & 7 & $3-43 \%$ & $7-100 \%$ & $7-100 \%$ \\
\hline & 3 PBTS, 1 PCS, 3 PEX & 0 PBTS, 1 PCS, 2 PEX & 3 PBTS, 1 PCS, 3 PEX & 3 PBTS, 1 PCS, 3 PEX \\
\hline \multirow{2}{*}{7} & 10 & $1-10 \%$ & $7-70 \%$ & $9-90 \%$ \\
\hline & 4 PBTS, 2 PCS, 4 PEX & 0 PBTS, 0 PCS, 1 PEX & 3 PBTS, 0 PCS, 4 PEX & 4 PBTS, 1 PCS, 4 PE \\
\hline \multirow{2}{*}{8} & 13 & $2-15 \%$ & $5-38 \%$ & $6-46 \%$ \\
\hline & 6 PBTS, 1 PCS, 6 PEX & 0 PBTS, 0 PCS, 2 PEX & 4 PBTS, 0 PCS, 1 PEX & 5 PBTS, 0 PCS, 1 PEX \\
\hline
\end{tabular}

atomic events being missed in association to a passenger resulting a composite event, PCS, being undetected. Overall, the sequence contains ten composite events which explains the value of $90 \%$ for $R$.

Sequence 8 performed most poorly with a value of $R=46 \%$. Interestingly, this was also the sequence for which $A$ was lowest at $62 \%$. The ground truth and the system output for this sequence are shown in the tables in Fig. 9.

Here we can see from the ground truth table that there are in total thirteen composite events: FBTS11 and PEX for person P1; MBTS19 and PEX for person 2; FBTS9 and PEX for person P3; MBTS18, PCS17, and PEX for person P4; FBTS2 and PEX for person P5; MBTS19 and PEX for person P6. However, only six of the events are correctly recognised, i.e. $R=6 / 13=46 \%$. In the case of the composite event PEX, only for person P6, it is correctly recognised; for others, the 

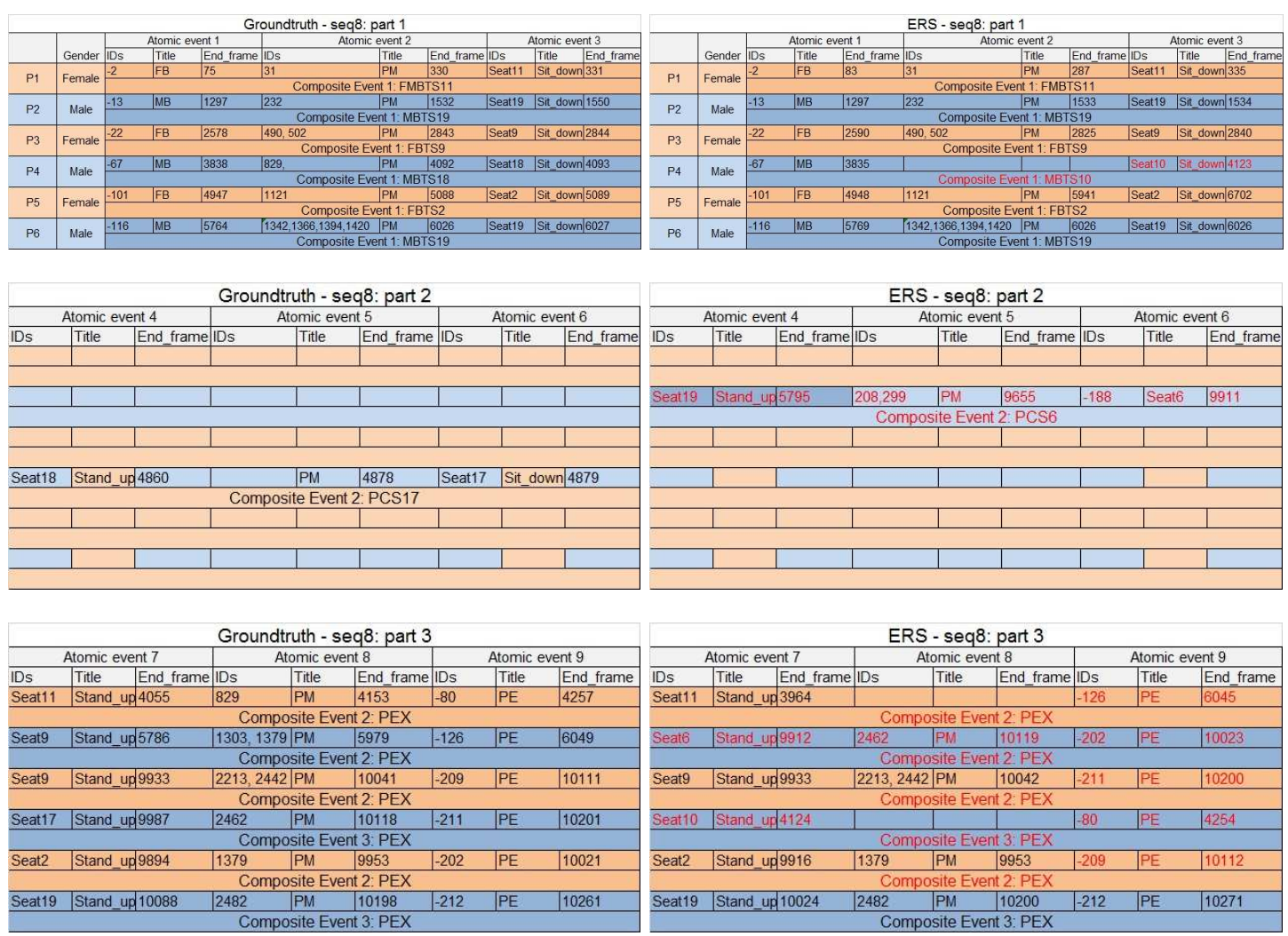

Figure 9: Ground truth and system output for sequence 8. (better viewed in colour)

composite event was mistakenly mixed up, that is the PEX of P1 was mistakenly assigned to P4, $\mathrm{P} 2$ to $\mathrm{P} 1, \mathrm{P} 3$ to $\mathrm{P} 5, \mathrm{P} 4$ to $\mathrm{P} 3$, and $\mathrm{P} 5$ to $\mathrm{P} 2$. Also for person P2 the composite event PCS6 was incorrectly recognised, namely the male was mistakenly recognised as sitting in seat 6 when in fact he had already exited the bus. The most serious mistakes were made on person P4. For the person the composite event MBTS18 was incorrectly recognised, the composite event PCS17 was not detected in addition to mistakenly assigned composite event PEX. To understand this, Table 6 shows a segment of eighteen atomic events that were detected: From table 6 we can see that event E127 is of type PM, in fact corresponding to the male moving up the gangway towards seat 18, and the female moving back to the exit and exiting the bus. On the left side of Fig. 10 are two images taken from sequence 8 which are a snapshot of the atomic event. Also shown in right side of Fig. 10, are the corresponding track on ground floor, for the PM event E127. 
Table 6: eighteen atomic events detected for sequence 8

\begin{tabular}{|l|l|l|r|r|r|r|r|r|}
\hline Event idx & Event ID & Event title & Start_frame & End_frame & StartX & StartY & EndX & EndY \\
\hline E121 & -67 & gender & 3835 & 3835 & 0 & 0 & 0 & 0 \\
\hline E122 & 786 & movement id 786 & 3839 & 3857 & -70 & 145 & -71 & 144 \\
\hline E123 & 796 & movement id 796 & 3879 & 3900 & -69 & 138 & -68 & 138 \\
\hline E124 & 797 & movement id 797 & 3880 & 3892 & 73 & 130 & 80 & 131 \\
\hline E125 & 808 & movement id 808 & 3911 & 3919 & 72 & 110 & 70 & 108 \\
\hline E126 & 826 & movement id 826 & 3954 & 3964 & 73 & 117 & 74 & 116 \\
\hline E127 & 829 & movement id 829 & 3962 & 4154 & 26 & -205 & 27 & -195 \\
\hline$\ldots$ & & & & & & & & \\
\hline E139 & -72 & Seat Sensor ON_10 & 4123 & 4123 & 0 & 0 & 0 & 0 \\
\hline
\end{tabular}
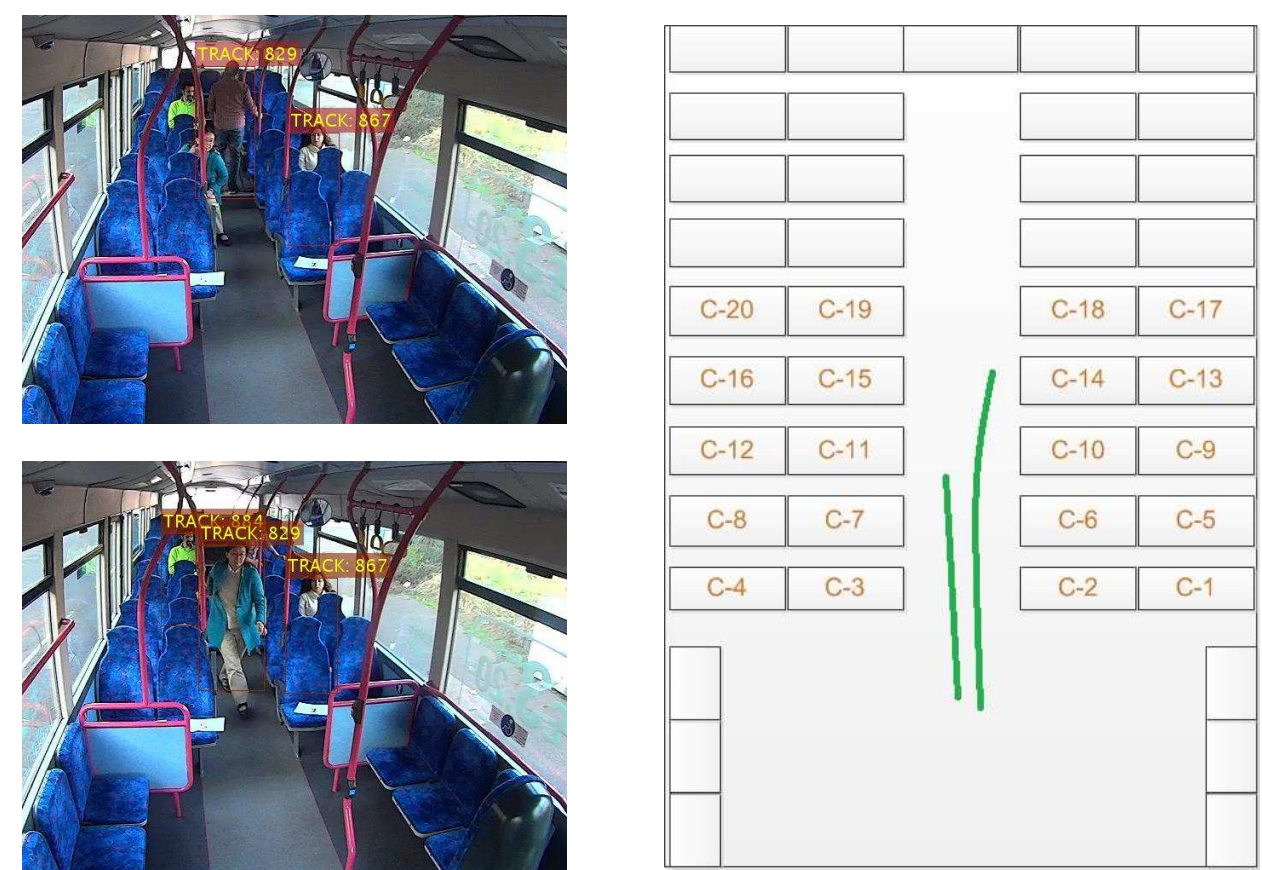

Figure 10: Sequence 8 - top-left: image of male moving close to seat 18; bottom-left: image of female moving away from seat 11, towards the bus door; right: trajectory corresponding to PM event E127 detected by a tracker - TRACK 829.

The partitioning at this point is as follows:

$\omega_{1}=\left\{e_{1}, \neg e_{2}, \neg e_{3}, e_{4}, \neg e_{5}, \neg e_{6}, \neg e_{7}, e_{8}, e_{9}, \neg e_{10}, \neg e_{11}, e_{12}, \neg e_{13}, e_{14}, \ldots, e_{16}\right.$, 


$$
\begin{gathered}
\neg e_{17}, e_{18}, \neg e_{19}, \ldots, \neg e_{22}, e_{23}, \neg e_{24}, \neg e_{25}, e_{26}, e_{27}, \neg e_{28}, \neg e_{29}, e_{30}, \ldots, e_{35}, \\
\neg e_{36}, e_{37}, \neg e_{38}, e_{39}, e_{40}, \neg e_{41}, \ldots, \neg e_{43}, e_{44}, \neg e_{45}, e_{46}, \neg e_{47}, e_{48}, e_{49}, \neg e_{50}, \ldots, \neg e_{76}, \\
e_{77}, e_{78}, \neg e_{79}, \ldots, \neg e_{86}, e_{87}, \neg e_{88}, e_{89}, \neg e_{90}, e_{91}, \neg e_{92}, e_{93}, e_{94}, e_{95}, \neg e_{96}, e_{97}, \\
\neg e_{98}, \neg e_{99}, e_{100}, \neg e_{101}, \ldots, \neg e_{105}, e_{106}, \neg e_{107}, e_{108}, \neg e_{109}, e_{110}, \neg e_{111}, \ldots, \neg e_{113}, \\
\left.e_{114}, \ldots, e_{117}, \neg e_{118}, e_{119},, \neg e_{120}, \ldots, \neg e_{123}, e_{124}, e_{125}, e_{126}\right\} \\
\omega_{2}=\left\{\neg e_{1}, \ldots, \neg e_{27}, e_{28}, e_{29}, \neg e_{30}, \ldots, \neg e_{46}, e_{47}, \neg e_{48}, \ldots, \neg e_{51}, e_{52}, e_{53}, \neg e_{54}, \ldots, \neg e_{67},\right. \\
\left.e_{68}, \neg e_{69}, \ldots, \neg e_{74}, e_{75}, \neg e_{76}, \ldots, \neg e_{126}\right\} \\
\omega_{3}=\left\{\neg e_{1}, \ldots, \neg e_{53}, e_{54}, e_{55}, e_{56}, \neg e_{57}, e_{58}, \neg e_{59}, e_{60}, \ldots, e_{67}, \neg e_{68}, \ldots, \neg e_{70},\right. \\
e_{71}, \ldots, e_{73}, \neg e_{74}, \ldots, \neg e_{81}, e_{82}, \neg e_{83}, \neg e_{84}, e_{85}, e_{86}, \neg e_{87}, \ldots, \neg e_{95}, \\
e_{96}, \neg e_{97}, \ldots, \neg e_{100}, e_{101}, e_{102}, \neg e_{103}, e_{104}, e_{105}, \neg e_{106}, \ldots, \neg e_{108}, e_{109}, \neg e_{110}, \\
\left.e_{111}, \ldots, e_{113}, \neg e_{114}, \ldots, \neg e_{119}, e_{120}, \neg e_{121}, e_{122}, e_{123}, \neg e_{124}, \ldots, \neg e_{126}\right\} \\
\omega_{4}=\left\{\neg e_{1}, \ldots, \neg e_{120}, e_{121}, \neg e_{122}, \ldots, \neg e_{126}\right\}
\end{gathered}
$$

When E127 is detected the system has ruled out partitions $\omega_{2}, \omega_{3}$ it should be assigned. As its starting half satisfies partition $\omega_{4}$, the ending half satisfies partition $\omega_{1}$, the system fails to assign it to any of them. Subsequently at E139 the system incorrectly assigns to $\omega_{4}$. Continue on, the system fails to correctly assign remaining events to partitions $\omega_{1}$ and $\omega_{4}$. Another similar mixed tracker at E214 (Fig. 11), a type of PM, corresponding to person P2 moving to the exit and person P5 staying on seat 2, results more mistakes in event association and consequently incorrectly recognised composite events. For this type of mixed-up atomic events, the system can not reason to correct assignments. However, when more atomic events are detected, if only the system can revise the beliefs of assigning them, previous incorrect partitions can possibly be corrected.

\subsection{Comparison}

A simple rule-based approach is chosen as the based line for comparison. In [? ] Ma et al. proposed a rule based approach to inferring events of interest by applying rules to combine existing events. Their method employs inference rules to capture new situations, than modifying custom code, hence ensuring a flexible solution for evolving situations. It was initially developed 


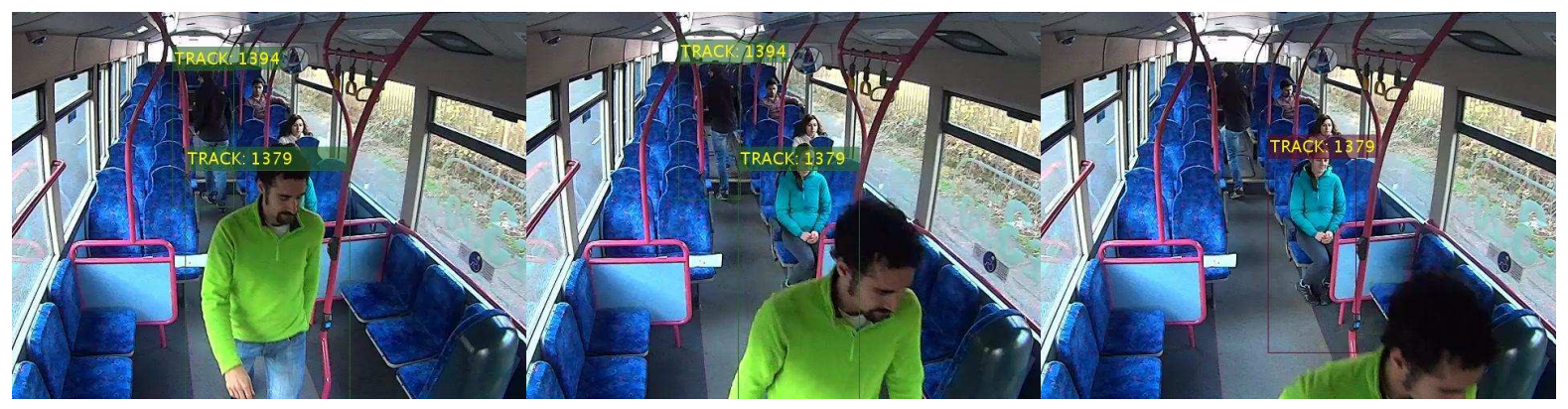

(a) frame 5966

(b) frame 5977

(c) frame 5982

Figure 11: Sequence 8 - instances of event E214 detected by a tracker - TRACK 1379.

for handling single subject scenarios. It was then adapted by the introduction of linking rules to work on multiple subject environments. The rules are used to link atomic events derived from video analytics by measuring the distance in space or time between two atomic events. Though their inference rules consider imprecision of atomic events derived from video anayltics, both inference rules and linking rules make the assumption that the occurrence of each atomic event can be observed, which is not always true considering imperfect video anayltics, in particular in a dynamic environment such as on a moving bus platform. When linking atomic events, their involvements in composite event inference are not considered at all. Our evidential reasoning approach powers with the functionality for handling these problems.

We employ DS theory to represent uncertainty in event modelling and event reasoning. DS theory is the generalisation of probability theory, which allows the representation of ignorance due to lack of knowledge. We compared our evidential approach with Bayesian approach, by adapting the evidential reasoning system with probabilities instead of mass functions, in recognising composite events from the set of atomic events having associated to a person. Bayesian approach lacks abilities of handling the problem of incomplete information in event reasoning.

The $R$ values obtained for each sequence with the rule-based approach and Bayesian approach are shown in the third and fourth columns respectively, together with those by our evidential reasoning system in the last columns, in Table 5. 


\section{Conclusions}

In this paper, we propose a novel approach for detection and recognition of composite events on video sequences where multiple subjects present. First, video-analytics and senor measurements are generated in the shape of events. Second, event association and composition are performed by combining the techniques of temporal relation representation, DS theory of evidence and hierarchical network modelling. Our approach can be used to correctly recognise composite events while separating atomic events of multiple subjects with the ability of handling the uncertainty in the video analytics.

Our framework has been evaluated on a real bus environment. The results show the promising performance of the proposed framework. Comprehensive tests on more video data collected from applications and comparison against state-of-art techniques are being performed as future work. 


\section{Appendix A. Constraint Rules}

Table A.1: List of rules for partitioning

\section{Golden rule: $\mathrm{RO}$}

Statement: An atomic event can not be carried out by more than one person.

Premise: $E_{i} . e T y p e \in\{P B, P M, P S I T, P S T D, P E\}$

Condition: $E_{i} \in S_{m} \wedge e_{i} \in \omega_{m}$

Result: $E_{i} \notin S_{n}, \neg e_{i} \in \omega_{n}, n \neq m$

\section{Constraint rule: $\mathbf{R} 1$}

Statement: If only one person presents in a period of time, all atomic events can only be undertaken by the person.

Premise: $E_{i} . e T y p e \in\{P B, P M, P S I T, P S T D, P E\}$

Condition: $S=S_{1} \wedge \Omega=\omega_{1}$

Result: $E_{i} \in S_{1}, e_{i} \in \omega_{1}$

\section{Constraint rule: $\mathbf{R} 2$}

Statement: A person can only aboard a bus once in a period of time.

Premise: $E_{i}$.eType $\in\{P B\} \wedge E_{j}$.eType $\in\{P B\}$

Condition: $E_{i} \in\left\{S_{m}\right\} \wedge e_{i} \in \omega_{m}$

Result: $E_{j} \notin S_{m}, \neg e_{j} \in \omega_{m}$

\section{Constraint rule: $\mathbf{R 3}$}

Statement: A person can only hold one track at a time

Premise: $E_{i}$. eType $\in\{P M\}, E_{j}$. eType $\in\{P M\}$

Condition: $E_{i}$.time ol $E_{j} . t i m e \wedge E_{i} \in S_{m} \wedge e_{i} \in \omega_{m}$

Result: $E_{j} \in S_{n}, e_{j} \in \omega_{n}, n \neq m$

\section{Constraint rule: $\mathbf{R} 4$}

Statement: One person can only appear at one place at a time.

Premise: $E_{i}$. eType, $E_{j}$. eType $\in\{P B, P M, P S I T, P S T D, P E\}$

Condition: $E_{i}$.location $\neq E_{j}$.location $\wedge E_{i}$.time ol $E_{j}$.time $\wedge E_{i} \in S_{m} \wedge e_{i} \in \omega_{m}$ 
Result: $E_{j} \in S_{n}, e_{j} \in \omega_{n}, n \neq m$

\section{Constraint rule: $\mathbf{R 5}$}

Statement: Two atomic events with the same object ID are carried out by a same person.

Premise: $E_{i}$. eType, $E_{j}$. eType $\in\{P B, P M, P S I T, P S T D, P E\}$

Condition: $E_{i} . o I D=E_{j} . o I D \wedge E_{i} \in S_{m} \wedge e_{i} \in \omega_{m}$

Result: $E_{j} \in S_{m}, e_{j} \in \omega_{m}$

\section{Constraint rule: $\mathbf{R 6}$}

Statement: Any atomic event happens before a person boards the bus is carried out by other persons.

Premise: $E_{i} . e T$ ype $\in P B, E_{j}$. eType $\in\{P B, P M, P S I T, P S T D, P E\}$

Condition: $E_{j}$.time $b E_{i}$.time $\wedge E_{i} \in S_{m}$

Result: $E_{j} \in S_{n}, e_{j} \in \omega_{n}, n \neq m$

\section{Constraint rule: $\mathbf{R 7}$}

Statement: Any atomic event happens after a person has exited the bus is carried out by other persons.

Premise: $E_{i}$. eType $\in P E, E_{j}$. T Type $\in\{P B, P M, P S I T, P S T D, P E\}$

Condition: $E_{j}$.time a $E_{i}$.time $\wedge E_{i} \in S_{m}$

Result: $E_{j} \in S_{n}, e_{j} \in \omega_{n}, n \neq m$

\section{Constraint rule: $\mathbf{R 8}$}

Statement: One person can not carry out two different atomic events at a time.

Premise: $E_{i}$.eType, $E_{j}$. eType $\in\{P B, P M, P S I T, P S T D, P E\}$

Condition: $E_{j}$.time ol $E_{i}$.time $\wedge E_{i}$.eType $\neq E_{j}$.eType $\wedge E_{i} \in S_{m}$

Result: $E_{j} \in S_{n}, e_{j} \in \omega_{n}, n \neq m$ 


\section{Appendix B. Case Study}

To help illustrate how our system works, we describe here an application scenario. In this scenario, two subjects, Alice and Bob, take a bus journey. The bus is a standard single deck bus in use by public transport in Northern Ireland. For recording, two cameras are deployed, one pointing at the front door of the bus, the other at the saloon.

Scenario 1. At a bus stop, Bob boards the bus and moves to a seat on a row in the middle of the bus saloon, and sits down (Fig. B1a). Alice boards the bus at the next stop and moves to a window seat on the first row, left-hand side, and sits down (Fig. B1b). Whilst Bob stands up and moves to the seat next to Alice and sits down (Fig. B1c). At the following stop, Alice stands up and moves to the door and alights the bus (Fig. B1d). Then Bob stands up and moves to the exit and exits the bus.

For the purposes of our application scenario, we are interested in the following atomic events: $\mathrm{PB}=$ "male or female boards bus", PM = "person moves from X to Y", PSIT = "person sits", PSTD = "person stands" and PE = "person exits". The composite events we want to infer from atomic events are: PBTS="person boards bus and transits to seat", PCS="person changes seat", and PEX="person exits bus".

\section{B1. Atomic Event Detection}

An atomic event $E$ is represented by tuple (eType, oID, date, time, location, source, reliaR, $v$ Frame, $m$ ) as in Definition 3. eType is the type of the atomic event, such as $P B$ and PM. oID is the identify number assigned by detection. date is the date on which the atomic event has detected. time is an interval of its starting time and ending time. location is the context of spaces the atomic event has covered. source shows which analytic module has provided the detection. reliaR is the reliability of the source. vFrame is the frame of discernment that holds all values that an atomic event of the type can have. For the four types of atomic events, we have

$$
\begin{array}{ll}
P B: & \text { vFrame }=\{M B, F B\} ; \\
P M: & \text { vFrame }=\{M S 1, \ldots, M S 20, M G W, M D R\} ;
\end{array}
$$




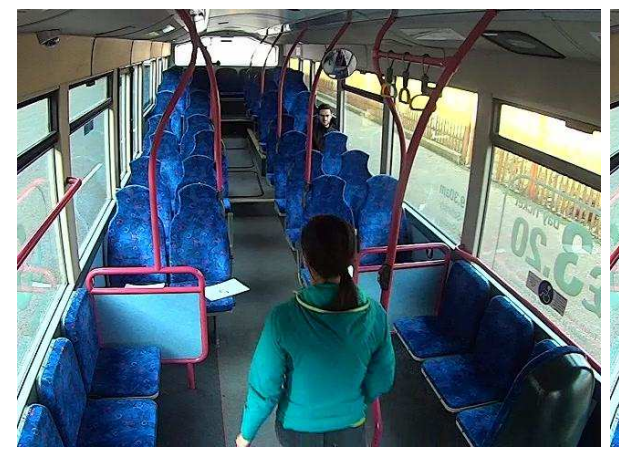

(a)

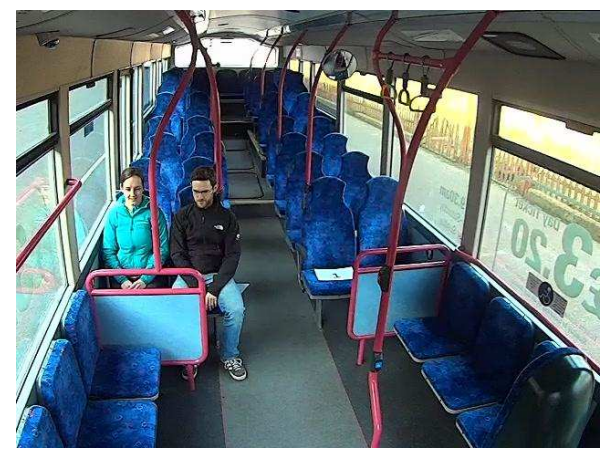

(c)

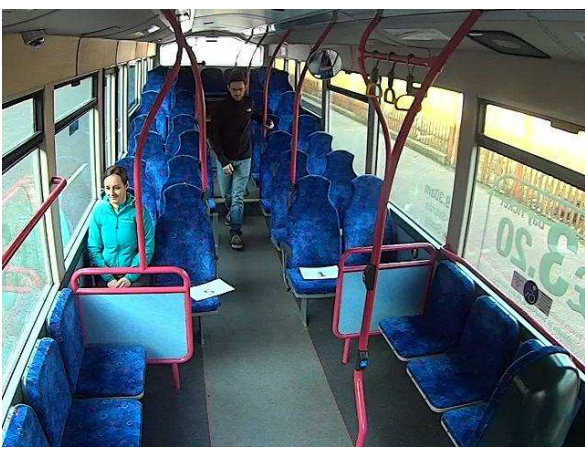

(b)

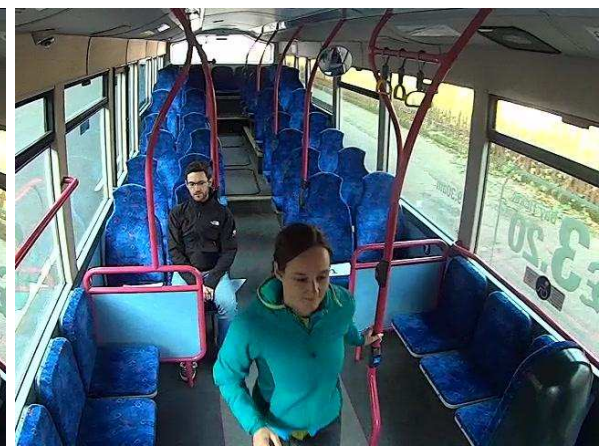

(d)

Figure B1: Four instances of the scenario sequence: (a) female enters (b) female seats and male stands up, moves (c) male and female seated (d) female exits

$$
\begin{array}{ll}
\text { PSIT: } & \text { vFrame }=\{S I T 1, \ldots, \text { SIT20, ᄀSIT }\} \\
\text { PSTD : } & \text { vFrame }=\{S T D 1, \ldots, S T D 20, \neg S T D\} ; \\
\text { PE : } & \text { vFrame }=\{E X, \neg E X\} .
\end{array}
$$

$m$ is the mass function obtained from a detection.

For the first type of atomic events we employ a camera pointing at the door of the bus. The well-known Jones and Viola face detector is then applied to the acquired video. The output of this is then input to a face-based gender classifier. The resulting output is the probability of the face as being either male or female. Thus, for example, we might have $p($ male $)=0.7$ and $p($ female $)=$ 0.3. Based on our training classification accuracy, the module is deemed to have a reliability of $r$ 
= 0.9. Thus, from Eq. 4 we obtain the corresponding mass distribution,

$$
\begin{aligned}
& m(\{\text { male }\})=0.7 * 0.9=0.63, m(\{\text { female }\})=0.3 * 0.9=0.27 \\
& m(\Theta)=1-m(\{\text { male }\})-m(\{\text { female }\})=0.1
\end{aligned}
$$

As the camera is pointing at the entrance, when we detect a male or female face in its field-of-view, we infer from this either MB or FB, respectively.

$$
m(\{M B\})=0.63, m(\{F B\})=0.27, m(\Theta)=0.1 .
$$

For the PM event we employ a $3 \mathrm{~d}$ tracker onto the acquired video from the camera pointing at the saloon of the bus. The output of the tracker is a trajectory from which we determine the startpoint and the end-point. We then calculate the distance from these points to several schematic locations nearby. These schematic locations consist of all seats, gangway, and door exit. We then use the distance of the tracker to a two closest schematic locations to calculate the mass values for the PM event. For example, for a tracker the distances of its endpoint to the two closest schematic locations, seat 5 and 6 , are calculated as $\operatorname{dist}($ seat 5$)=78$ and $\operatorname{dist}($ seat 6 , gangway) $=26$. The corresponding mass functions are then given by

$$
\begin{aligned}
& m(\{M S 5\})=26 / 104 * 0.8=0.2, \\
& m(\{M S 6, M G W\})=78 / 104 * 0.8=0.6, m(\Theta)=0.2
\end{aligned}
$$

where the reliability of 0.8 is derived from the accuracy measurements of the tracker as reported in [?].

For the PSIT and PSTD events a 3D tracker is used to estimate the shoulder level of an individual in real world space. The resulting output is sitting if the shoulder level goes below a threshold, otherwise standing. PSIT and PSTD are paired together. That means, for example, if there is a SIT9, there should be a STD9 afterwards. Based on our training accuracy, the module is deemed to have a reliability of $r=0.9$. Thus, from Eq. 4 we obtain the corresponding mass distribution,

$$
m(\{S I T 9\})=1.0 * 0.9=0.9, m(\Theta)=1-m(\{S I T 9\})=0.1 .
$$


From the outputs of video analytics, twenty-six atomic events are detected for the sequence of scenario 1. The details of oID, eType, time in the format of an interval [Start frame, End frame], and mass function $m$, are given in Table B.1. For simplicity, the details of date, location, source, reliaR are not listed. $v$ Frame has given at the beginning of this sub-section.

Table B.1: List of atomic events

\begin{tabular}{|c|c|c|c|c|c|}
\hline Event & oID & eType & Start frame & End frame & Mass function \\
\hline E1 & -2 & PB & 55 & 55 & $m(\{M B\})=0.81, m(\Theta)=0.19$ \\
\hline E2 & 3 & PM & 194 & 259 & $m(\{M S 14\})=0.43, m(\{M S 11, M G W\})=0.37, m(\Theta)=0.2$ \\
\hline E3 & -4 & $\mathrm{~PB}$ & 769 & 769 & $m(\{M B\})=0.09, m(\{F B\})=0.81, m(\Theta)=0.1$ \\
\hline E4 & 14 & PM & 894 & 906 & $m(\{M D R\})=0.72, m(\{M S 3, M G W\})=0.08, m(\Theta)=0.2$ \\
\hline E5 & -5 & PSIT & 896 & 896 & $m(\{S I T 2\})=0.9, m(\Theta)=0.1$ \\
\hline E6 & -6 & PSTD & 927 & 927 & $m(\{S T D 2\})=0.9, m(\Theta)=0.1$ \\
\hline E7 & -7 & PSIT & 948 & 948 & $m(\{S I T 2\})=0.9, m(\Theta)=0.1$ \\
\hline E8 & 18 & PM & 948 & 950 & $m(\{M S 4\})=0.64, m(\{M S 3, M G W\})=0.16, m(\Theta)=0.2$ \\
\hline E9 & -8 & PSIT & 950 & 950 & $m(\{S I T 4\})=0.9, m(\Theta)=0.1$ \\
\hline E10 & 18 & $\mathrm{PM}$ & 950 & 1062 & $m(\{M S 4\})=0.45, m(\{M S 8\})=0.35, m(\Theta)=0.2$ \\
\hline E11 & 20 & PM & 961 & 1062 & $m(\{M S 3, M G W\})=0.62, m(\{M S 4\})=0.18, m(\Theta)=0.2$ \\
\hline E12 & -9 & PSTD & 977 & 977 & $m(\{S T D 2\})=0.9, m(\Theta)=0.1$ \\
\hline E13 & -11 & PSIT & 1062 & 1062 & $m(\{S I T 3\})=0.9, m(\Theta)=0.1$ \\
\hline E14 & 18 & $\mathrm{PM}$ & 1062 & 1361 & $m(\{M D R\})=0.55, m(\{M S 3, M G W\})=0.25, m(\Theta)=0.2$ \\
\hline E15 & 20 & PM & 1062 & 1359 & $m(\{M S 3, M G W\})=0.58, m(\{M S 4\})=0.22, m(\Theta)=0.2$ \\
\hline E16 & 44 & PM & 1170 & 1184 & $m(\{M S 7, M G W\})=0.64, m(\{M S 8\})=0.16, m(\Theta)=0.2$ \\
\hline E17 & -13 & PSTD & 1359 & 1359 & $m(\{S T D 4\})=0.9, m(\Theta)=0.1$ \\
\hline E18 & 20 & PM & 1359 & 1577 & $m(\{M D R\})=0.54, m(\{M S 3, M G W\})=0.26, m(\Theta)=0.2$ \\
\hline E19 & -15 & $\mathrm{PE}$ & 1370 & 1370 & $m(\{E X\})=0.8, m(\Theta)=0.2$ \\
\hline E20 & 66 & PM & 1428 & 1438 & $m(\{M S 9\})=0.47, m(\{M S 13\})=0.33, m(\Theta)=0.2$ \\
\hline E21 & 68 & $\mathrm{PM}$ & 1445 & 1455 & $m(\{M S 9\})=0.49, m(\{M S 13\})=0.31, m(\Theta)=0.2$ \\
\hline E22 & -16 & PSIT & 1448 & 1448 & $m(\{S I T 5\})=0.9, m(\Theta)=0.1$ \\
\hline E23 & 82 & $\mathrm{PM}$ & 1516 & 1531 & $m(\{M S 9\})=0.45, m(\{M S 13\})=0.35, m(\Theta)=0.2$ \\
\hline E24 & -19 & PSTD & 1578 & 1578 & $m(\{S T D 5\})=0.9, m(\Theta)=0.1$ \\
\hline E25 & -18 & PSTD & 1578 & 1578 & $m(\{S T D 3\})=0.9, m(\Theta)=0.1$ \\
\hline E26 & -20 & $\mathrm{PE}$ & 1586 & 1586 & $m(\{E X\})=0.8, m(\Theta)=0.2$ \\
\hline
\end{tabular}




\section{B2. Evidential Event Networks}

Three categories of composite events are concerned: Male/Female Boards bus and Transits to Seat x(PBTS: MBTS/FBTS), Person Changes Seat (PCS), Person EXits bus (PEX). Composite events are consisted of atomic events. For the case study, we can construct three evidential event networks: $E E N_{P B T S}, E E N_{P C S}$ and $E E N_{P E X}$, presenting the hierarchical structures of the composite events with their atomic events. Fig. B2a B2c illustrate three EEN respectively.

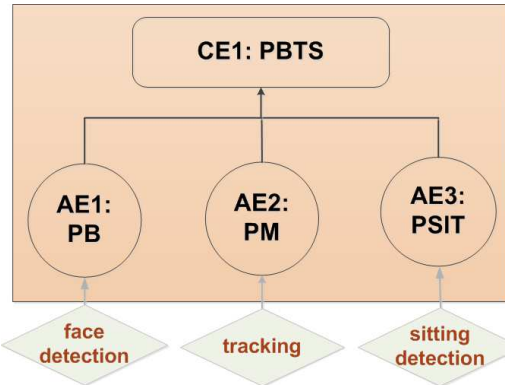

(a) PBTS

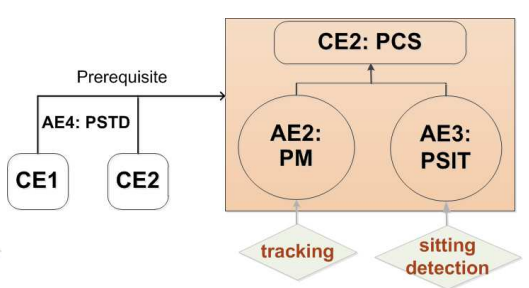

(b) PCS

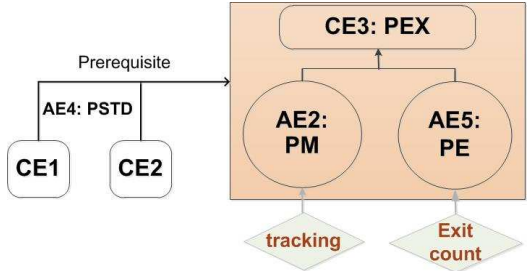

(c) PEX

Figure B2: Three evidential event networks

By Definition 5, we have $E E N_{P B T S}=\left(N D_{P B T S}, E G_{P B T S}, M M_{P B T S}\right), E E N_{P C S}=\left(N D_{P C S}, E G_{P C S}\right.$, $\left.M M_{P C S}\right)$, and $E E N_{P e X}=\left(N D_{P E X}, E G_{P E X}, M M_{P E X}\right) . N D$ is a set of event nodes, $N D_{P B T S}==\{A E 1$, $A E 2, A E 3, C E 1\}, N D_{P C S}=\{A E 2, A E 3, C E 2\}, N D_{P E X}=\{A E 2, A E 4, C E 3\}$. An atomic event node is same as an atomic event in B1, except that it has attribute pID indicating to whom it concerns, level telling it is an atomic event (or a composite event for a composite event node). For eample, $A E 1 . p I D=1, A E 1$. level $=$ 'atomic'. For a composite event node, its date is same as its children at the atomic level, and its time interval is decided by the start time of the first child node and the end time of the last child node. oID, location source and reliaR are not required for an composite event node. For the case study, the details of atomic events have been given above. The frame of discernment for a composite event node is as follows.

$$
\begin{array}{cl}
\text { PBTS : } & \text { vFrame }=\{\text { MBTS } 1, \ldots, \text { MBTS } 20, \text { MBTGW, } \\
& \text { FBTS } 1, \ldots, \text { FBTS } 20, F B T G W, \neg P B T S\} \\
\text { PCS : } & \text { vFrame }=\{P C S 1, \ldots, \text { PCS } 20, \neg P C S\}
\end{array}
$$




$$
\text { PEX : } \quad \text { vFrame }=\{P E X, \neg P E X\}
$$

Each arc of $E G$ in an evidential event network represents the relationship between one node to another, which can be represented by a multivalued mapping in $M M$. Table B.2 shows the multivalued mappings for the case study.

Table B.2: List of multi-valued mappings

\begin{tabular}{|l|l|}
\hline Relationship & multivalued mapping \\
\hline$A E 1 \rightarrow C E 1$ & $\Gamma(\{M B\})=\{M B T S 1, \ldots, M B T S 20, M B T G W\}$, \\
& $\Gamma(\{F B\})=\{F B T S 1, \ldots, F B T S 20, F B T G W\}, \Gamma\left(\Theta_{A E 1}\right)=\Theta_{C E 1}$ \\
\hline$A E 2 \rightarrow C E 1$ & $\Gamma(\{M S 1\})=\{M B T S 1, F B T S 1\}, \ldots \Gamma(\{M S 20\})=\{M B T S 20, F B T S 20\}$, \\
& $\Gamma(\{M G W\})=\{M B T G W, F B T G W\}, \Gamma(\{M D R\})=\{\neg P B T S\}, \Gamma\left(\Theta_{A E 2}\right)=\Theta_{C E 1}$ \\
\hline$A E 3 \rightarrow C E 1$ & $\Gamma(\{S I T 1\})=\{M B T S 1, F B T S 1\}, \ldots \Gamma(\{S I T 20\})=\{M B T S 20, F B T S 20\}$, \\
& $\Gamma(\{\neg S I T\})=\{\neg P B T S\}, \Gamma\left(\Theta_{A E 3}\right)=\Theta_{C E 1}$ \\
\hline$A E 2 \rightarrow C E 2$ & $\Gamma(\{M S 1\})=\{P C S 1\}, \ldots \Gamma(\{M S 20\})=\{P C S 20\}$, \\
& $\Gamma(\{M G W\})=\{\neg P C S\}, \Gamma(\{M D R\})=\{\neg P C S\}, \Gamma\left(\Theta_{A E 2}\right)=\Theta_{C E 2}$ \\
\hline$A E 3 \rightarrow C E 2$ & $\Gamma(\{S I T 1\})=\{P C S 1\}, \ldots \Gamma(\{S I T 20\})=\{P C S 20\}$, \\
& $\Gamma(\{\neg S I T\})=\{\neg P C S\}, \Gamma\left(\Theta_{A E 3}\right)=\Theta_{C E 2}$ \\
\hline$A E 2 \rightarrow C E 3$ & $\Gamma(\{M S 1\})=\{\neg P E X\}, \ldots \Gamma(\{M S 20\})=\{\neg P E X\}$, \\
& $\Gamma(\{M G W\})=\{\neg P E X\}, \Gamma(\{M D R\})=\{P E X\}, \Gamma\left(\Theta_{A E 2}\right)=\Theta_{C E 3}$ \\
\hline$A E 4 \rightarrow C E 3$ & $\Gamma(\{E X\})=\{P E X\}, \Gamma(\{\neg E X\})=\{\neg P E X\}, \Gamma\left(\Theta_{A E 4}\right)=\Theta_{C E 3}$ \\
\hline
\end{tabular}

\section{B3. Atomic Event Association}

Now twenty-six derived atomic events are going to be partitioned into two groups, which are associated to two passengers respectively. Let $\Xi=\left\{E_{1}, \ldots, E_{26}\right\}$ and $\Omega=\left\{e_{1}, \neg e_{1}, \ldots, e_{26}, \neg e_{26}\right\}$. The goal of event association is to have $S=S_{1} \cup S_{2}, S_{1} \cap S_{2}=\emptyset$, that also means to have $\omega_{1} \subset \Omega$ and $\omega_{2} \subset \Omega$, satisfying Proposition 1 . The association goes through: partitioning $\Xi$ by applying the constraint rules, and if more than two partitionings arises, optimisation by selecting the most probable partitioning with a minimum conflict factor.

With domain knowledge, we have constraints to guide the association of the atomic events. The specific constraints being applied to the scenario example are listed in Table A.1 of Appendix 
A.

\section{Stage 1 - Partitioning}

Start from $E_{1}$ until $E_{26}$; Golden Rule R0 always applies;

(1-2) $E_{1}$. eType $=P B, E_{2}$. eType $=P M$

Condition: $S=S_{1}$

Apply: R1

Results: $e_{1}, e_{2} \in \omega_{1}$

Partitioning:

$$
\omega_{1}=\left\{e_{1}, e_{2}, e_{3}, \neg e_{3}, \ldots, e_{26}, \neg e_{26}\right\}
$$

(3) $E_{3}$. eType $=P B$

Condition: $E_{3} . e T y p e=P B ;\left(e_{1}, e_{2}\right) \in \omega_{1}$

Apply: R2 and R6

Results: initialise $\omega_{2}=\Omega, e_{3} \in \omega_{2}, \neg e_{3} \in \omega_{1} ;\left(\neg e_{1}, \neg e_{2}\right) \in \omega_{2}$

Partitioning:

$$
\begin{aligned}
& \omega_{1}=\left\{e_{1}, e_{2}, \neg e_{3}, e_{4}, \neg e_{4}, \ldots, e_{26}, \neg e_{26}\right\} \\
& \omega_{2}=\left\{\neg e_{1}, \neg e_{2}, e_{3}, e_{4}, \neg e_{4}, \ldots, e_{26}, \neg e_{26}\right\}
\end{aligned}
$$

(4) $E_{4}$. Type $=P M$

Condition: $\omega=\omega_{1} \cup \omega_{2} ; E_{4} . e T y p e=P M, E_{4}$.location $-E_{2}$.location $>\tau_{\text {location }}, E_{4}$.location $E_{3}$.location $<\tau_{\text {location }}, e_{2} \in \omega_{1}, e_{3} \in \omega_{2}$

Apply: R3

Results: $\neg e_{4} \in \omega_{1}, e_{4} \in \omega_{2}$

Partitioning:

$$
\begin{aligned}
& \omega_{1}=\left\{e_{1}, e_{2}, \neg e_{3}, \neg e_{4}, e_{5}, \neg e_{5}, \ldots, e_{26}, \neg e_{26}\right\} \\
& \omega_{2}=\left\{\neg e_{1}, \neg e_{2}, e_{3}, e_{4}, e_{5}, \neg e_{5}, \ldots, e_{26}, \neg e_{26}\right\}
\end{aligned}
$$


(5) $E_{5}$. eType $=$ PSIT

Condition: $\omega=\omega_{1} \cup \omega_{2} ; E_{5}$. eType $=$ PS IT, E. $E_{5}$ location $-\left(E_{2}, E_{4}\right)$.location $>\tau_{\text {location }}$, $e_{2} \in \omega_{1}, e_{4} \in \omega_{2}$

Apply: R4

Results: $\neg e_{5} \in\left(\omega_{1}, \omega_{2}\right)$

Partitioning:

$$
\begin{aligned}
& \omega_{1}=\left\{e_{1}, e_{2}, \neg e_{3}, \neg e_{4}, \neg e_{5}, e_{6}, \neg e_{6}, \ldots, e_{26}, \neg e_{26}\right\} \\
& \omega_{2}=\left\{\neg e_{1}, \neg e_{2}, e_{3}, e_{4}, \neg e_{5}, e_{6}, \neg e_{6}, \ldots, e_{26}, \neg e_{26}\right\}
\end{aligned}
$$

(6) $E_{6}$. eType $=P S T D$

Condition: $\omega=\omega_{1} \cup \omega_{2} ; E_{6}$. eType $=$ PSTD, $E_{6}$.location $=E_{5}$.location, $e_{5} \notin\left(\omega_{1}, \omega_{2}\right)$

Apply: R4

Results: $\neg e_{6} \in\left(\omega_{1}, \omega_{2}\right)$

Partitioning:

$$
\begin{aligned}
& \omega_{1}=\left\{e_{1}, e_{2}, \neg e_{3}, \neg e_{4}, \neg e_{5}, \neg e_{6}, e_{7}, \neg e_{7}, \ldots, e_{26}, \neg e_{26}\right\} \\
& \omega_{2}=\left\{\neg e_{1}, \neg e_{2}, e_{3}, e_{4}, \neg e_{5}, \neg e_{6}, e_{7}, \neg e_{7}, \ldots, e_{26}, \neg e_{26}\right\}
\end{aligned}
$$

(7) $E_{7}$. eType $=$ PSIT

Condition: same as in (5)

Apply: R4

Results: $\neg e_{7} \in\left(\omega_{1}, \omega_{2}\right)$

Partitioning:

$$
\begin{aligned}
& \omega_{1}=\left\{e_{1}, e_{2}, \neg e_{3}, \neg e_{4}, \neg e_{5}, \neg e_{6}, \neg e_{7}, e_{8}, \neg e_{8}, \ldots, e_{26}, \neg e_{26}\right\} \\
& \omega_{2}=\left\{\neg e_{1}, \neg e_{2}, e_{3}, e_{4}, \neg e_{5}, \neg e_{6}, \neg e_{7}, e_{8}, \neg e_{8}, \ldots, e_{26}, \neg e_{26}\right\}
\end{aligned}
$$

(8) $E_{8}$. eType $=P M$

Condition: $\omega=\omega_{1} \cup \omega_{2} ; E_{8} . e T y p e=P M, E_{8}$.location $-E_{2}$. location $>\tau_{\text {location }}, E_{8}$. location $E_{4}$. location $<\tau_{\text {location }}, e_{2} \in \omega_{1}, e_{4} \in \omega_{2}$ 
Apply: R4

Results: $\neg e_{8} \in \omega_{1}, e_{8} \in \omega_{2}$

Partitioning:

$$
\begin{aligned}
& \omega_{1}=\left\{e_{1}, e_{2}, \neg e_{3}, \neg e_{4}, \neg e_{5}, \ldots, \neg e_{8}, e_{9}, \neg e_{9}, \ldots, e_{26}, \neg e_{26}\right\} \\
& \omega_{2}=\left\{\neg e_{1}, \neg e_{2}, e_{3}, e_{4}, \neg e_{5}, \neg e_{6}, \neg e_{7}, e_{8}, e_{9}, \neg e_{9}, \ldots, e_{26}, \neg e_{26}\right\}
\end{aligned}
$$

(9) $E_{9}$. eType $=$ PS IT

Condition: $\omega=\omega_{1} \cup \omega_{2} ; E_{8}$. eType $=P M, E_{9}$. eType $=$ PSIT, E . $_{8}$ time $=E_{9}$. time, $E_{8}$. location $=E_{9}$. location $; \neg e_{8} \in \omega_{1}, e_{8} \in \omega_{2}$

Apply: R4

Results: $\neg e_{9} \in \omega_{1}, e_{9} \in \omega_{2}$

Partitioning:

$$
\begin{aligned}
& \omega_{1}=\left\{e_{1}, e_{2}, \neg e_{3}, \ldots, \neg e_{9}, e_{10}, \neg e_{10}, \ldots, e_{26}, \neg e_{26}\right\} \\
& \omega_{2}=\left\{\neg e_{1}, \neg e_{2}, e_{3}, e_{4}, \neg e_{5}, \neg e_{6}, \neg e_{7}, e_{8}, e_{9}, e_{10}, \neg e_{10}, \ldots, e_{26}, \neg e_{26}\right\}
\end{aligned}
$$

(10) $E_{10}$. eType $=P M$

Condition: $\omega=\omega_{1} \cup \omega_{2} ; E_{8} . o I D=E_{10} . o I D, \neg e_{8} \in \omega_{1}, e_{8} \in \omega_{2}$

Apply: R5

Results: $\neg e_{10} \in \omega_{1}, e_{10} \in \omega_{2}$

Partitioning:

$$
\begin{aligned}
& \omega_{1}=\left\{e_{1}, e_{2}, \neg e_{3}, \ldots, \neg e_{10}, e_{11}, \neg e_{11}, \ldots, e_{26}, \neg e_{26}\right\} \\
& \omega_{2}=\left\{\neg e_{1}, \neg e_{2}, e_{3}, e_{4}, \neg e_{5}, \neg e_{6}, \neg e_{7}, e_{8}, e_{9}, e_{10}, e_{11}, \neg e_{11}, \ldots, e_{26}, \neg e_{26}\right\}
\end{aligned}
$$

(11) $E_{11}$. eType $=P M$

Condition: $\omega=\omega_{1} \cup \omega_{2} ; E_{11}$.eType $=P M ; E_{2}$.eType $=P M, E_{2}$. location $=E_{11}$.location; $E_{10}$. T Type $=$ PM, $E_{10}$.time ol $E_{11}$.time $; e_{2} \in \omega_{1}, e_{10} \in \omega_{2}$ Apply: R4 and R5 
Results: $e_{11} \in \omega_{1}, \neg e_{11} \in \omega_{2}$

Partitioning:

$$
\begin{aligned}
& \omega_{1}=\left\{e_{1}, e_{2}, \neg e_{3}, \ldots, \neg e_{10}, e_{11}, e_{12}, \neg e_{12}, \ldots, e_{26}, \neg e_{26}\right\} \\
& \omega_{2}=\left\{\neg e_{1}, \neg e_{2}, e_{3}, e_{4}, \neg e_{5}, \neg e_{6}, \neg e_{7}, e_{8}, e_{9}, e_{10}, \neg e_{11}, e_{12}, \neg e_{12}, \ldots, e_{26}, \neg e_{26}\right\}
\end{aligned}
$$

(12) $E_{12}$. eType $=P S T D$

Condition: same as in (6)

Apply: same as in (6)

Results: $\neg e_{12} \in \omega_{1}, \neg e_{12} \in \omega_{2}$

Partitioning:

$\omega_{1}=\left\{e_{1}, e_{2}, \neg e_{3}, \ldots, \neg e_{10}, e_{11}, \neg e_{12}, e_{13}, \neg e_{13}, \ldots, e_{26}, \neg e_{26}\right\}$

$\omega_{2}=\left\{\neg e_{1}, \neg e_{2}, e_{3}, e_{4}, \neg e_{5}, \neg e_{6}, \neg e_{7}, e_{8}, e_{9}, e_{10}, \neg e_{11}, \neg e_{12}, e_{13}, \neg e_{13}, \ldots, e_{26}, \neg e_{26}\right\}$

(13) $E_{13}$. eType $=$ PS IT

Condition: $\omega=\omega_{1} \cup \omega_{2} ; E_{11}$. eType $=P M, E_{13}$. eType $=$ PSIT, E $E_{11}$.time $=E_{13}$. time, $E_{11}$.location $=E_{13}$.location $; e_{11} \in \omega_{1}, \neg e_{11} \in \omega_{2}$

Apply: R4

Results: $e_{13} \in \omega_{1}, \neg e_{13} \in \omega_{2}$

Partitioning:

$$
\begin{gathered}
\omega_{1}=\left\{e_{1}, e_{2}, \neg e_{3}, \ldots, \neg e_{10}, e_{11}, \neg e_{12}, e_{13}, e_{14}, \neg e_{14}, \ldots, e_{26}, \neg e_{26}\right\} \\
\omega_{2}=\left\{\neg e_{1}, \neg e_{2}, e_{3}, e_{4}, \neg e_{5}, \neg e_{6}, \neg e_{7}, e_{8}, e_{9}, e_{10}, \neg e_{11}, \neg e_{12}, \neg e_{13},\right. \\
\left.e_{14}, \neg e_{14}, \ldots, e_{26}, \neg e_{26}\right\}
\end{gathered}
$$

(14) $E_{14}$. eType $=P M$

Condition: same as in (10)

Apply: R5

Results: $\neg e_{14} \in \omega_{1}, e_{14} \in \omega_{2}$ 
Partitioning:

$$
\begin{aligned}
\omega_{1}= & \left\{e_{1}, e_{2}, \neg e_{3}, \ldots, \neg e_{10}, e_{11}, \neg e_{12}, e_{13}, \neg e_{14}, e_{15}, \neg e_{15}, \ldots, e_{26}, \neg e_{26}\right\} \\
\omega_{2}= & \left\{\neg e_{1}, \neg e_{2}, e_{3}, e_{4}, \neg e_{5}, \neg e_{6}, \neg e_{7}, e_{8}, e_{9}, e_{10}, \neg e_{11}, \neg e_{12}, \neg e_{13},\right. \\
& \left.e_{14}, e_{15}, \neg e_{15}, \ldots, e_{26}, \neg e_{26}\right\}
\end{aligned}
$$

(15) $E_{15}$. eType $=P M$

Condition: $\omega=\omega_{1} \cup \omega_{2} ; E_{15} . o I D=E_{11} . o I D, e_{11} \in \omega_{1}, \neg e_{11} \in \omega_{2}$

Apply: R5

Results: $e_{15} \in \omega_{1}, \neg e_{15} \in \omega_{2}$

Partitioning:

$$
\begin{gathered}
\omega_{1}=\left\{e_{1}, e_{2}, \neg e_{3}, \ldots, \neg e_{10}, e_{11}, \neg e_{12}, e_{13}, \neg e_{14}, e_{15}, e_{16}, \neg e_{16}, \ldots, e_{26}, \neg e_{26}\right\} \\
\omega_{2}=\left\{\neg e_{1}, \neg e_{2}, e_{3}, e_{4}, \neg e_{5}, \neg e_{6}, \neg e_{7}, e_{8}, e_{9}, e_{10}, \neg e_{11}, \neg e_{12}, \neg e_{13},\right. \\
\left.e_{14}, \neg e_{15}, e_{16}, \neg e_{16}, \ldots, e_{26}, \neg e_{26}\right\}
\end{gathered}
$$

(16) $E_{16}$. Type $=P M$

Condition: $\omega=\omega_{1} \cup \omega_{2} ;\left(E_{14}, E_{15}, E_{16}\right)$. eType $=P M,\left(E_{14}, E_{15}\right)$.time ol $E_{16}$.time, $e_{15} \in \omega_{1}$, $e_{14} \in \omega_{2}$

Apply: R3

Results: $\neg e_{16} \in\left(\omega_{1}, \omega_{2}\right)$

Partitioning:

$$
\begin{aligned}
\omega_{1}= & \left\{e_{1}, e_{2}, \neg e_{3}, \ldots, \neg e_{10}, e_{11}, \neg e_{12}, e_{13}, \neg e_{14}, e_{15}, \neg e_{16}, e_{17}, \neg e_{17}, \ldots, e_{26}, \neg e_{26}\right\} \\
\omega_{2}= & \left\{\neg e_{1}, \neg e_{2}, e_{3}, e_{4}, \neg e_{5}, \neg e_{6}, \neg e_{7}, e_{8}, e_{9}, e_{10}, \neg e_{11}, \neg e_{12}, \neg e_{13},\right. \\
& \left.e_{14}, \neg e_{15}, \neg e_{16}, e_{17}, \neg e_{17}, \ldots, e_{26}, \neg e_{26}\right\}
\end{aligned}
$$

(17) $E_{17 . e T y p e}=P S T D$

Condition: $\omega=\omega_{1} \cup \omega_{2} ; E_{17}$. eType $=$ PSTD, E $E_{9}$. Type $=$ PSIT, E $E_{17}$.location = E9.location, $\neg e_{9} \in \omega_{1}, e_{9} \in \omega_{2}$ 
Apply: R4

Results: $\neg e_{17} \in \omega_{1}, e_{17} \in \omega_{2}$

Partitioning:

$\omega_{1}=\left\{e_{1}, e_{2}, \neg e_{3}, \ldots, \neg e_{10}, e_{11}, \neg e_{12}, e_{13}, \neg e_{14}, e_{15}, \neg e_{16}, \neg e_{17}, e_{18}, \neg e_{18}, \ldots, e_{26}, \neg e_{26}\right\}$

$\omega_{2}=\left\{\neg e_{1}, \neg e_{2}, e_{3}, e_{4}, \neg e_{5}, \neg e_{6}, \neg e_{7}, e_{8}, e_{9}, e_{10}, \neg e_{11}, \neg e_{12}, \neg e_{13}, e_{14}\right.$,

$\left.\neg e_{15}, \neg e_{16}, e_{17}, e_{18}, \neg e_{18}, \ldots, e_{26}, \neg e_{26}\right\}$

(18) $E_{18}$. eType $=P M$

Condition: same as in (15)

Apply: R5

Results: $e_{18} \in \omega_{1}, \neg e_{18} \in \omega_{2}$

Partitioning:

$$
\begin{gathered}
\omega_{1}=\left\{e_{1}, e_{2}, \neg e_{3}, \ldots, \neg e_{10}, e_{11}, \neg e_{12}, e_{13}, \neg e_{14}, e_{15}, \neg e_{16}, \neg e_{17}, e_{18},\right. \\
\left.e_{19}, \neg e_{19}, \ldots, e_{26}, \neg e_{26}\right\} \\
\omega_{2}=\left\{\neg e_{1}, \neg e_{2}, e_{3}, e_{4}, \neg e_{5}, \neg e_{6}, \neg e_{7}, e_{8}, e_{9}, e_{10}, \neg e_{11}, \neg e_{12}, \neg e_{13},\right. \\
\left.e_{14}, \neg e_{15}, \neg e_{16}, e_{17}, \neg e_{18}, e_{19}, \neg e_{19}, \ldots, e_{26}, \neg e_{26}\right\}
\end{gathered}
$$

(19) $E_{19 .}$. Type $=P E$

Condition: $\omega=\omega_{1} \cup \omega_{2} ; E_{19}$. eType $=P E, E_{18}$. eType $=P M, E_{18}$.time ol $E_{19}$. time,

$E_{17}$. Type $=P M, E_{17}$.location $-E_{19}$. location $<\tau_{\text {location }} ; e_{18} \in \omega_{1}, e_{17} \in \omega_{2}$

Apply: R4

Results: $\neg e_{19} \in \omega_{1}, e_{19} \in \omega_{2}$

Partitioning:

$$
\begin{gathered}
\omega_{1}=\left\{e_{1}, e_{2}, \neg e_{3}, \ldots, \neg e_{10}, e_{11}, \neg e_{12}, e_{13}, \neg e_{14}, e_{15}, \neg e_{16}, \neg e_{17},\right. \\
\left.e_{18}, \neg e_{19}, e_{20}, \neg e_{20}, \ldots, e_{26}, \neg e_{26}\right\} \\
\omega_{2}=\left\{\neg e_{1}, \neg e_{2}, e_{3}, e_{4}, \neg e_{5}, \neg e_{6}, \neg e_{7}, e_{8}, e_{9}, e_{10}, \neg e_{11}, \neg e_{12}, \neg e_{13},\right. \\
\left.e_{14}, \neg e_{15}, \neg e_{16}, e_{17}, \neg e_{18}, e_{19}, e_{20}, \neg e_{20}, \ldots, e_{26}, \neg e_{26}\right\}
\end{gathered}
$$


(20) $E_{20}$. eType $=P M$

Condition: $\omega=\omega_{1} \cup \omega_{2} ; E_{20}$. eType $=P M, E_{18}$. eType $=P M, E_{18}$. time ol $E_{20}$. time, $E_{19}$. Type $=P E ; e_{18} \in \omega_{1}, e_{19} \in \omega_{2}$

Apply: R3 and R7

Results: $\neg e_{20} \in\left(\omega_{1}, \omega_{2}\right)$

Partitioning:

$$
\begin{gathered}
\omega_{1}=\left\{e_{1}, e_{2}, \neg e_{3}, \ldots, \neg e_{10}, e_{11}, \neg e_{12}, e_{13}, \neg e_{14}, e_{15}, \neg e_{16}, \neg e_{17}, e_{18},\right. \\
\left.\neg e_{19}, \neg e_{20}, e_{21}, \neg e_{21}, \ldots, e_{26}, \neg e_{26}\right\} \\
\omega_{2}=\left\{\neg e_{1}, \neg e_{2}, e_{3}, e_{4}, \neg e_{5}, \neg e_{6}, \neg e_{7}, e_{8}, e_{9}, e_{10}, \neg e_{11}, \neg e_{12}, \neg e_{13},\right. \\
\left.e_{14}, \neg e_{15}, \neg e_{16}, e_{17}, \neg e_{18}, e_{19}, \neg e_{20}, e_{21}, \neg e_{21}, \ldots, e_{26}, \neg e_{26}\right\}
\end{gathered}
$$

$(21-24)\left(E_{21}, E_{23}\right) . e T y p e=P M, E_{22} . e T y p e=P S I T, E_{24} . e T y p e=P S T D$

Condition: same as in (20)

Apply: R3 and R7

Results: $\left(\neg e_{20}, \ldots, \neg e_{24}\right) \in\left(\omega_{1}, \omega_{2}\right)$

Partitioning:

$$
\begin{gathered}
\omega_{1}=\left\{e_{1}, e_{2}, \neg e_{3}, \ldots, \neg e_{10}, e_{11}, \neg e_{12}, e_{13}, \neg e_{14}, e_{15}, \neg e_{16}, \neg e_{17}, e_{18},\right. \\
\left.\neg e_{19}, \ldots, \neg e_{24}, e_{25}, \neg e_{25}, e_{26}, \neg e_{26}\right\} \\
\omega_{2}=\left\{\neg e_{1}, \neg e_{2}, e_{3}, e_{4}, \neg e_{5}, \neg e_{6}, \neg e_{7}, e_{8}, e_{9}, e_{10}, \neg e_{11}, \neg e_{12}, \neg e_{13},\right. \\
\left.\quad e_{14}, \neg e_{15}, \neg e_{16}, e_{17}, \neg e_{18}, e_{19}, \neg e_{20}, \ldots, \neg e_{24}, e_{25}, \neg e_{25}, e_{26}, \neg e_{26}\right\}
\end{gathered}
$$

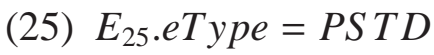

Condition: $\omega=\omega_{1} \cup \omega_{2} ; E_{25}$. eType $=$ PSTD, $E_{13}$. eType $=$ PSIT, $E_{25}$. location $=$ $E_{13}$.location, $e_{13} \in \omega_{1}, E_{19} . e T y p e=P E, e_{19} \in \omega_{2}$

Apply: R4 and R7

Results: $e_{25} \in \omega_{1}, \neg e_{25} \in \omega_{2}$

Partitioning:

$$
\omega_{1}=\left\{e_{1}, e_{2}, \neg e_{3}, \ldots, \neg e_{10}, e_{11}, \neg e_{12}, e_{13}, \neg e_{14}, e_{15}, \neg e_{16}, \neg e_{17}, e_{18},\right.
$$




$$
\begin{gathered}
\left.\neg e_{19}, \ldots, \neg e_{24}, e_{25}, e_{26}, \neg e_{26}\right\} \\
\omega_{2}=\left\{\neg e_{1}, \neg e_{2}, e_{3}, e_{4}, \neg e_{5}, \neg e_{6}, \neg e_{7}, e_{8}, e_{9}, e_{10}, \neg e_{11}, \neg e_{12}, \neg e_{13},\right. \\
\left.e_{14}, \neg e_{15}, \neg e_{16}, e_{17}, \neg e_{18}, e_{19}, \neg e_{20}, \ldots, \neg e_{25}, e_{26}, \neg e_{26}\right\}
\end{gathered}
$$

(26) $E_{26}$. eType $=P E$

Condition: $\omega=\omega_{1} \cup \omega_{2} ; E_{26}$. eType $=P E, E_{25}$. eType $=$ PSTD, $E_{25}$. location $-E_{26}$. location $<$ $\tau_{\text {location }}, e_{25} \in \omega_{1}, E_{19}$. eType $=P E, e_{19} \in \omega_{2}$

Apply: R7

Results: $e_{26} \in \omega_{1}, \neg e_{26} \in \omega_{2}$

Partitioning:

$$
\begin{gathered}
\omega_{1}=\left\{e_{1}, e_{2}, \neg e_{3}, \ldots, \neg e_{10}, e_{11}, \neg e_{12}, e_{13}, \neg e_{14}, e_{15}, \neg e_{16}, \neg e_{17}, e_{18},\right. \\
\left.\neg e_{19}, \ldots, \neg e_{24}, e_{25}, e_{26}\right\} \\
\omega_{2}=\left\{\neg e_{1}, \neg e_{2}, e_{3}, e_{4}, \neg e_{5}, \neg e_{6}, \neg e_{7}, e_{8}, e_{9}, e_{10}, \neg e_{11}, \neg e_{12}, \neg e_{(} 13\right), \\
\left.e_{14}, \neg e_{15}, \neg e_{16}, e_{17}, \neg e_{18}, e_{19}, \neg e_{20}, \ldots, \neg e_{26}\right\}
\end{gathered}
$$

In this scenario, there is no multiple partitionings raised. Therefore, the optimisation does not apply.

The final results of atomic event association are as follows.

$$
\begin{gathered}
S=S_{1} \cup S_{2}, \\
S_{1}=\left\{E_{1}, E_{2}, E_{11}, E_{13}, E_{15}, E_{18}, E_{25}, E_{26}\right\}, \\
S_{2}=\left\{E_{3}, E_{4}, E_{8}, E_{9}, E_{10}, E_{14}, E_{17}, E_{19}\right\} . \\
\omega=\omega_{1} \cup \omega_{2}, \\
\omega_{1}=\left\{e_{1}, e_{2}, \neg e_{3}, \ldots, \neg e_{10}, e_{11}, \neg e_{12}, e_{13}, \neg e_{14},\right. \\
\left.e_{15}, \neg e_{16}, \neg e_{17}, e_{18}, \neg e_{19}, \ldots, \neg e_{24}, e_{25}, e_{26}\right\}, \\
\omega_{2}=\left\{\neg e_{1}, \neg e_{2}, e_{3}, e_{4}, \neg e_{5}, \ldots, \neg e_{7}, e_{8}, e_{9}, e_{10},\right. \\
\neg e_{11}, \ldots, \neg e_{13}, e_{14}, \neg e_{15}, \neg e_{16}, e_{17}, \neg e_{18}, e_{19}, \\
\left.\neg e_{20}, \ldots, \neg e_{26}\right\} .
\end{gathered}
$$




\section{B4. Composite Event Recognition}

Now the atomic events associated to a passenger are going to be transferred to the evidential event networks and to infer the composite events.

Passenger 1 has associated with the atomic event set $\left\{E_{1}, E_{2}, E_{11}, E_{13}, E_{15}, E_{18}, E_{25}, E_{26}\right\}$. $E_{2}$. TType $=P M, E_{11}$. eType $=P M, E_{2} . m_{\text {end }} \cap E_{11} . m_{\text {start }}=M S 14, E_{11}$. starttime $\gg E_{2}$. endtime, $E_{2}$. startlocation $\neq E_{2}$.endlocation, $E_{25}$.eType $=P S T D, E_{11}$.startlocation $\neq E_{11}$.endlocation, therefore $E_{11}$ indicates that a composite event ends and another starts. $E_{25}$.eType $=$ PSTD, $E_{25}$ is used as a point that ends a composite event and starts another composite event. $E_{15}$ and $E_{18}$ take place between $E_{13}$ and $E_{25}$, their evidence support $E_{13}$ staying at seat 3. Thus $E_{15}$ and $E_{18}$ don't contribute to inference of the composite events.

$E_{1}$ and $E_{2}$ become the nodes at the lower-level in the network $E E N_{P B T S}$ as shown in Fig.B2a, are used to infer the composite event $C E 1: P B T S$ as the node at the higher-level. $E_{26}$ is in the network $E E N_{P E X}$, Fig.B2c, and is going to infer $C E 3: P E X$.

The inference of composite event $C E 1$ starts at translating the mass functions of the nodes at the lower-level into the node at the higher-level, and then combine these together. On the combined mass function, BetP on each single element is calculated. The final decision is made on the element with the highest $\operatorname{BetP}$.

On the event network CE1: PBTS,

(i) $m_{E_{1}}$ and $m_{E_{2}}$ are transferred onto $C E 1$ by using Eq. 2 and applying the multivalued mappings in Table B.2. Therefore, we have $m_{1}$ and $m_{2}$ along vacuous $m_{3}$ representing no knowledge on node AE3, as follows.

$$
\begin{aligned}
& m_{1}(\{M B T S 1, \ldots \ldots ., \operatorname{MBTS} 20, \operatorname{MBTGW}\})=m_{E_{1}}(\{M B\})=0.81 ; m_{1}(\Theta)=m_{E_{1}}(\Theta)=0.19 . \\
& m_{2}(\{M B T S 14, \text { FBTS } 14\})=m_{E_{2}}(\{M S 14\})=0.43 ; \\
& m_{2}(\{M B T S 11, M B T G W, F B T S 11, F B T G W\})=m_{E_{2}}(\{M S 11, M G W\})=0.37 \\
& m_{2}(\Theta)=m_{E_{2}}(\Theta)=0.2 . \\
& m_{3}(\Theta)=m_{E_{3}}(\Theta)=1 .
\end{aligned}
$$


(ii) Combining $\left(m_{1} \oplus m_{2}\right) \oplus m_{3}$ by Eq. 3, We have $m$ :

$$
\begin{aligned}
& m(\{M B T S 14\})=0.35 ; m(\{M B T S 11, M T G W\})=0.30 ; \\
& m(\{M B T S 1, \ldots, M B T S 20, M B T G W\})=0.16 ; m(\{M B T S 14, F B T S 14\})=0.08 ; \\
& m(\{M B T S 11, M B T G W, F B T S 11, F B T G W\})=0.07 ; m(\Theta)=0.04 .
\end{aligned}
$$

(iii) From $m$, we can calculate BetP by Eq. 5:

$$
\begin{aligned}
& \operatorname{Bet} P(\{M B T S 14\})=0.40 ; \operatorname{Bet} P(\{M B T S 11\})=0.18 \\
& \operatorname{BetP}(\{F B T S 14\})=0.04 ; \operatorname{BetP}(\{F B T S 11\})=0.02 .
\end{aligned}
$$

With the highest $\operatorname{BetP}(\{M B T S 14\})$, we reach the decision that composite event MBTS14: the male boards the bus and transits to sit on seat 14 , is inferred.

On the event network $C E 2: P C S, E_{11}$ and $E_{13}$ are used to infer $C E 2$. The same steps are gone throught to reach the decision that composite event PCS3: the person changes to seat 3, with $\operatorname{Bet} P(\{P C S 3\})=0.92$, is inferred.

On the event network $C E 3: P E X, E_{25}$ as $A E 5$ is used to infer $C E 3$. The decision is that composite event PEX: the person exits the bus with $\operatorname{BetP}(\{P E X\})=0.9$, is inferred.

The same procedure applies to passenger 2 with the associated atomic event set $\left\{E_{3}, E_{4}, E_{8}\right.$, $\left.E_{9}, E_{10}, E_{14}, E_{17}, E_{19}\right\}$. The composite events inferred are FBTS4: the female boards the bus and transits to sit on seat 4, PEX: the person exits the bus. 


\section{Appendix C. Bus Sequences}

The first sequence presents a normal bus journey and consists of a male and female boarding the bus, moving into the saloon to a seat and sitting down. After a short period they stand up, move back down the gangway and exit. Fig. C1 shows the example frames of sequence 1.

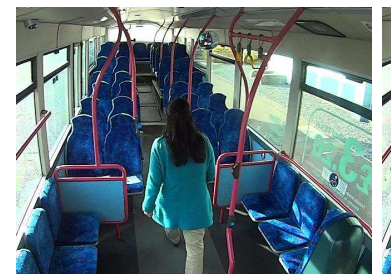

(a) frame 190

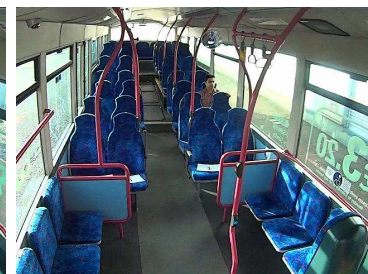

(b) frame 1450

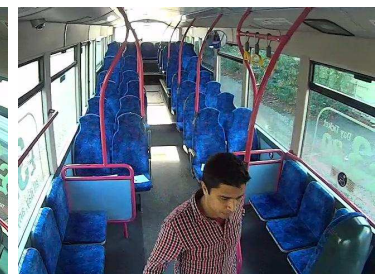

(c) frame 2304

Figure $\mathrm{C} 1$ : Example frames of sequence 1

Sequences 2-3 present a journey in which a passenger changes seat whilst the bus is moving. This is unusual and is indicative of a passenger who may feel threatened or one who is trying to threaten another passenger. These consist of a male and female entering the saloon and then moving along the gangway to seats and sitting down. After a short period one of them stands and moves to a different seat. At the next bus stop, both passengers stand up and move back down the gangway and exit the bus. With sequence 3, the example frames of the scenario are illustrated in Fig. C2. it is worthy to point our that Sequence 2 is used to interpret case studies in the appendices (Appendix B).

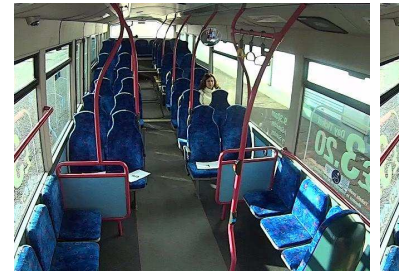

(a) frame 1133

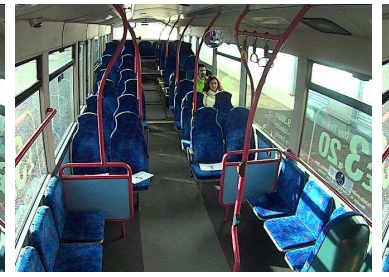

(b) frame 1458

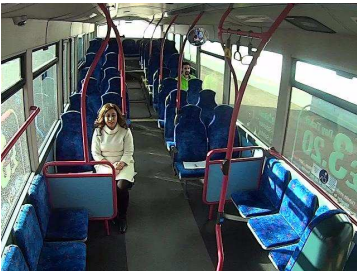

(c) frame 1672

Figure C2: Example frames of sequence 3

Sequence 4 presents a type of threatening behaviour in which one passenger loiters near another who is seated. At a bus stop, a female passenger boards and moves to a seat and sits down. 
The male at the next stop enters and moves to beside the seat occupied by the female passenger and loiters in the gangway. At the following stop, the female passenger stands up and moves to the exit and exits. The male passenger then follows and moves to the exit and eventually exits. Fig. C3 shows the example frames of sequence 4 .

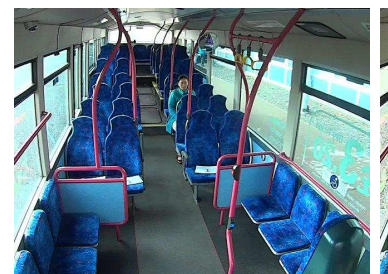

(a) frame 459

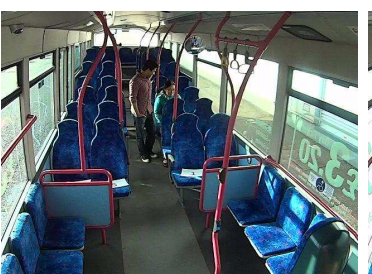

(b) frame 1426

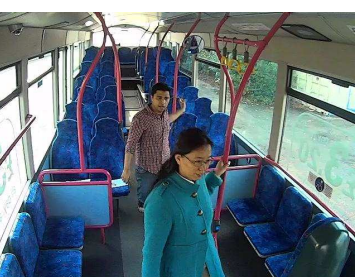

(c) frame 2423

Figure C3: Example frames of sequence 4

Sequence 5 presents a more threatening behaviour in which both passengers change seat. In this sequence the female passenger enters the bus and moves to seat and sits down. At a different stop, the male passenger enters the bus and moves to the seat right behind the female passenger and sits down. The female passenger then stands up and moves to a different seat and sits down. The male passenger stands up and moves to the seat beside the female and sits down. The female passenger stands up and moves to the exit and exits the bus. The male stands up and moves to the exit and exits the bus. The example frames of the scenario are shown in Fig. C4.

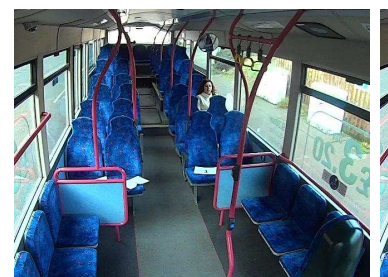

(a) frame 351

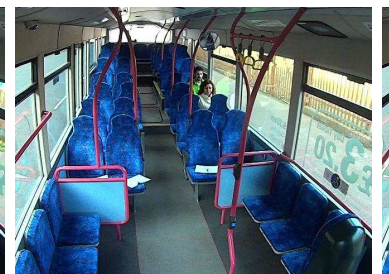

(b) frame 1110

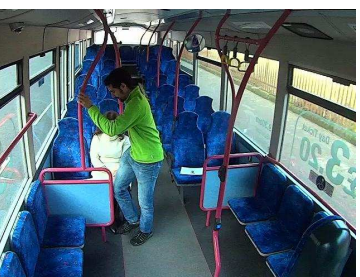

(c) frame 1380

Figure C4: Example frames of sequence 5

Sequence 6 consists of three passengers, 2 male and 1 female. Fig. C5 shows the example frames of the sequence. In this experiment, the female passenger enters and moves to seat $\mathrm{C}$ 10 and sits down. The first male passenger enters and moves nearby seat C-10 and loiters in 
the gangway. The female passenger stands up and moves to seat C-3 and sits down. The male passenger sits down on seat C-10, previously occupied by the female passenger. The second male passenger then boards, and moves to beside seat C-3 and loiters in the gangway. The female stands up, moves to the exit and exits the bus. The second male then sits down on seat C-3, vacated by the female passenger. The first male stands up and moves to the exit and exits the bus. Afterwards, the second male passenger stands up and moves to the exit and exits the bus.

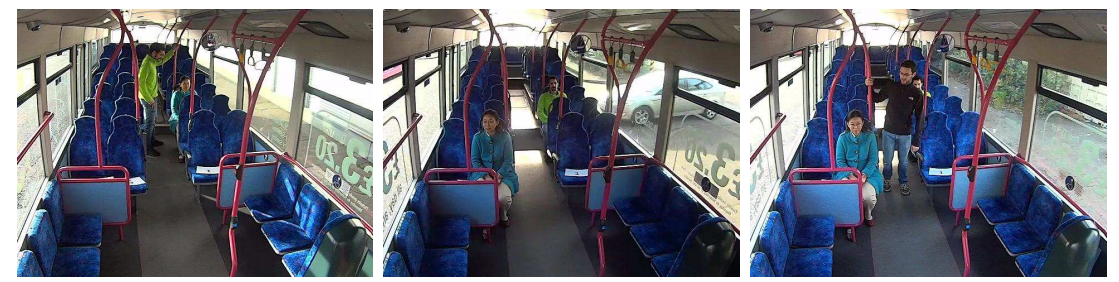

(a) frame 1500

(b) frame 2196

(c) frame 2659

Figure C5: Example frames of sequence 6

Sequence 7 presents a complicated sequence consisting of two male and female passengers with several seat changes and loitering incidents. The scenario is illustrated with the example frames shown in Fig. C6. In this sequence, the first male passenger boards at the first bus stop and moves to seat C-19 and sits down. At the second bus stop, the first female passenger boards and moves to seat C-9 and sits down. At the third stop, the second male passenger boards and moves to the gangway, beside seat C-9, and loiters. The first male passenger stands and moves to gangway. The second male moves to seat $\mathrm{C}-19$ vacated by the first male passenger and sits down. The first male passenger moves to seat $\mathrm{C}-1$ and sits down. At the fourth stop, the second female passenger boards, moves to seat C-2 and sits down. She then stands, moves to seat C-3 and sits down. At the next stop, the first male passenger stands, moves to the exit and exits the bus. The second male passenger stands, moves to the exit and exits the bus. At the last bus stop, the second female passenger then stands, moves to the exit and exits the bus. Lastly, the first female stands, moves to the exit and exits the bus.

The final sequence, 8 , is the most complicated one with six people involved, three each of male and female gender. This again involves several seat changes and loitering incidents, and also 


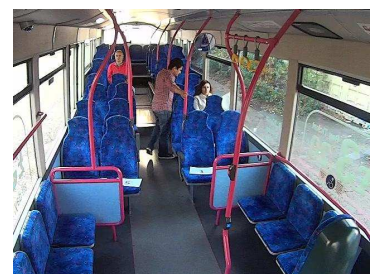

(a) frame 2748

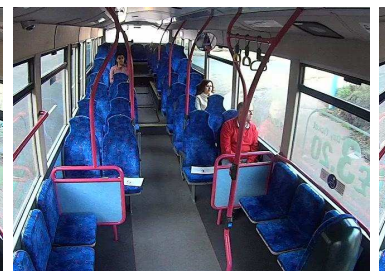

(b) frame 3688

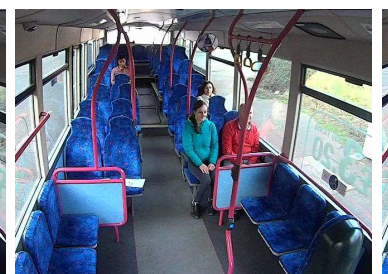

(c) frame 4041

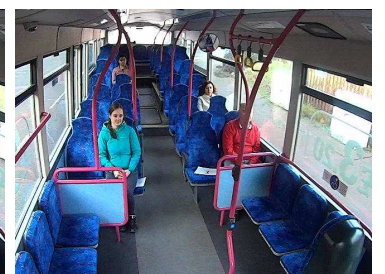

(d) frame 4187

Figure C6: Example frames of sequence 7

consists of two passenger passing each other in the gangway. The first female passenger boards, moves to seat $\mathrm{C}-11$ and sits down. At the next stop, the first male passenger boards, moves to seat C-19 and sits down. At the following stop, the second female passenger boards, moves to seat C-9 and sits down. At the fourth stop, the second male passenger boards and moves along the gangway. Meanwhile, the first female passenger stands and exits the bus, and the second male passenger sits down on seat C-18. At the following stop, the third female passenger boards the bus, moves to seat $\mathrm{C}-2$ and sits down. The second male passenger moves to the window seat $\mathrm{C}-17$. At the sixth stop, the third male passenger boards and moves to the gangway. At the same time, the first male passenger stands and passes the third male passenger in the gangway. The first male passenger exits the bus and the third male sits down on seat C-19. At the last stop, the third female stands and exits the bus. The second female moves to the exit and exits the bus, and the second male moves to the gangway. The third male stands. The second male exits the bus, and the third male moves to the gangway, then the exit and exits the bus. Fig. C7 shows the example frames in this video sequence.

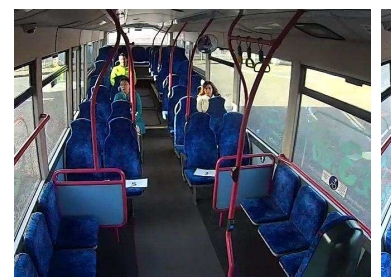

(a) frame 3358

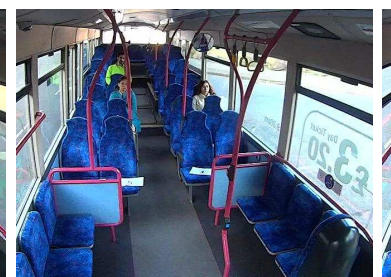

(b) frame 3554

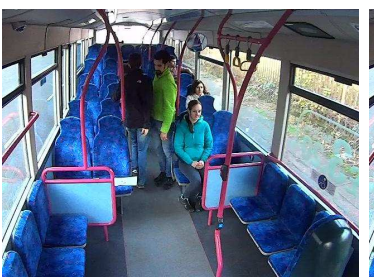

(c) frame 5917

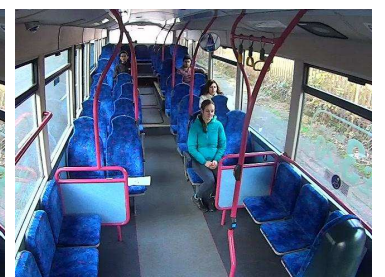

(d) frame 6054

Figure C7: Example frames of sequence 8 


\section{Acknowledgements}

This work is partially supported by the CSIT project funded by UK EPSRC under the grant EP/H049606/1, Invest NI and various industrial partners. We would like to thank Fabian CampbellWest and Bhargav Mitra for preparing video data, Niall McLaughlin for his valuable discussions. Thanks also go to the researchers and $\mathrm{PhD}$ students in the group for giving their time to participate in the experiments.

\section{References}

\section{References}

[1] T. Moeslund, A. Hilton, V. Kruger, A survey of advances in vision-based human motion capture and analysis, Computer Vision and Image Understanding 104 (2006) 90-126.

[2] D. Weinland, R. Ronfard, E. Boyer, A survey of vision-based methods for action representation, segmentation and recognition, Computer Vision and Image Understanding 115 (2011) 224-241.

[3] R. Poppe, A survey on vision-based human action recognition, Image and Vision Computing 28 (2010) 976-990.

[4] O. Popoola, K. Wang, Video-based abnormal human behavior recognition-a review, IEEE Transactions on Systems, Man, and Cybernetics, Part C: Applications and Reviews 42 (2012) 865-878.

[5] R. Turaga, P.and Chellappa, V. Subrahmanian, O. Udrea, Machine recognition of human activities: A survey, IEEE Transactions on Circuits, Systems and Video Teachnology 18 (2008) 1473-1488.

[6] G. Lavee, E. Rivlin, M. Rudzsky, Understanding video events: A survey of methods for automatic interpretation of semantic occurrences in video, IEEE Transactions on Systems, Man, and Cybernetics, Part C: Applications and Reviews 39 (5) (2009) 489-504.

[7] A. D. Newton, Crime on public transport, Encyclopedia of Criminology and Criminal Justice (2014) 709-720.

[8] T. deCampos, A survey on computer vision tools for action recognition, crowd surveillance and suspect retrieval, in: XXXIV Congresso da Sociedade Brasileira de Computacao (CSBC), 2014, pp. 1123-1132.

[9] S. Hongeng, R. Nevatia, Multi-agent event recognition, in: Procs. of ICCV, 2001, pp. 84-91.

[10] I. Atmosukarto, B. Ghanem, N. Ahuja, Trajectory-based fisher kernel representation for action recognition in videos., in: Procs. of ICPR, 2012, pp. 3333-3336.

[11] D. Ramanan, D. Forsyth, A. Zisserman, Tracking people by learning their appearance, IEEE Transactions on Pattern Analysis and Machine Intelligence 29 (1) (2007) 65-81.

[12] F. Bashir, A. Khokhar, D. Schonfeld, Object trajectory-based activity classification and recognition using hidden markov models, IEEE Transactions on Image Processing 16 (2007) 1912-1919. 
[13] J. Shotton, A. Fitzgibbon, M. Cook, T. Sharp, M. Finocchio, R. Moore, A. Kipman, A. Blake, Real-time human pose recognition in parts from single depth images, in: Procs. of CVPR, 2011, pp. 1297-1304.

[14] H. Zhou, H. Hu, H. Liu, J. Tang, Classification of upper limb motion trajectory using shape features, IEEE Transactions on System, Man, and Cybern. - Part C 42 (6) (2012) 970-982.

[15] L. Bourdev, J. Malik, Poselets: body part detectors trained using 3d human pose annotations, in: Procs. of ICCV, 2009, pp. 1365-1372.

[16] A. Yao, J. Gall, G. Fanelli, L. Gool, Does human action recognition benefit from pose estimation?, in: Procs. of BMVC, 2011.

[17] A. Kläser, M. Marszalek, C. Schmid, A spatio-temporal descriptor based on 3d-gradients, in: Procs. of BMVC, 2008, pp. 995-1004.

[18] Y. Ke, R. Sukthankar, M. Hebert, Event detection in crowded videos, in: Procs. of ICCV, 2007, pp. 1-8.

[19] H. Wang, A. Kläser, C. Schmid, C.-L. Liu, Action recognition by dense trajectories, in: Procs. of CVPR, 2011, pp. 3169-3176.

[20] D. Oneata, J. Verbeek, C. Schmid, Efficient Action Localization with Approximately Normalized Fisher Vectors, in: Procs. of CVPR, 2014.

[21] S. Sadanand, J. J. Corso, Action bank: A high-level representation of activity in video., in: Procs. of CVPR, 2012, pp. 1234-1241.

[22] Y.-L. Tian, R. Feris, A. Hampapur, Real-Time Detection of Abandoned and Removed Objects in Complex Environments, in: Procs. of The Eighth International Workshop on Visual Surveillance, 2008.

[23] Q. Fan, P. Gabbur, S. Pankanti, Relative attributes for large-scale abandoned object detection, in: Procs. of ICCV, 2013, pp. 2736-2743.

[24] J. Jacques-Jr, S. Mussef, C. Jung, Crowd Analysis Using Computer Vision Techniques, IEEE Signal Processing Magazine 27 (2010) 66-77.

[25] H. Idrees, N. Warner, M. Shah, Tracking in dense crowds using prominence and neighborhood motion concurrence, Image Vision Comput. 32 (1) (2014) 14-26.

[26] B. Zhou, X. Wang, X. Tang, Understanding collective crowd behaviors: Learning a mixture model of dynamic pedestrian-agents., in: Procs. of CVPR, 2012, pp. 2871-2878.

[27] S. Yi, X. Wang, C. Lu, J. Jia, L0 regularized stationary time estimation for crowd group analysis, in: Procs. of CVPR, 2014.

[28] M. Leach, E. Sparks, N. Robertson, Contextual anomaly detection in crowded surveillance scenes, Pattern Recognition Letters 44 (2014) 71-79.

[29] S. Cho, H. Kang, Abnormal behavior detection using hybrid agents in crowded scenes, Pattern Recognition Letters 44 (2014) 64-70.

[30] J. Kittler, W. Christmas, T. deCampos, D. Windridge, F. Yan, J. Illingworth, M. Osman, Domain anomaly 
detection in machine perception: A system architecture and taxonomy, IEEE Transactions on Pattern Analysis and Machine Intelligence 36 (5) (2014) 845-859.

[31] G. Lavee, M. Rudzsky, E. Rivlin, Propagating certainty in petri nets for activity recognition, IEEE Transactions on Circuits and Systems for Video Technology 23 (2) (2013) 326-337.

[32] J. Chen, Y. Cui, G. Ye, D. Liu, S.-F. Chang, Event-driven semantic concept discovery by exploiting weakly tagged internet images, in: Procs. of International Conference on Multimedia Retrieval, 2014, pp. 1:1-1:8.

[33] W. Li, Q. Yu, H. Sawhney, N. Vasconcelos, Recognizing activities via bag of words for attribute dynamics., in: Procs. of CVPR, IEEE, 2013, pp. 2587-2594.

[34] N. Chomsky, Syntactic Structures, Mouton, 1957.

[35] C. Petri, Communication with automata, Tech. Rep. AD0630125, Defense Tech. Inf. Cntr. (1966).

[36] M. Ryoo, J. Aggarwal, Recognition of composite human activities through context-free grammar based representation, in: Procs. of CVPR, 2006, pp. 1709-1718.

[37] G. Lavee, A. Borzin, E. Rivlin, M. Rudzsky, Building petri nets from video event ontologies, in: Procs. of ISVC, Springer-Verlag Berlin Heidelberg, 2007, pp. 442-451.

[38] S. Guler, J. Burns, A. Hakeem, Y. Sheikh, M. Shah, M. Thonnat, F. Bremond, N. Maillot, T. Vu, I. Haritaoglu, R. Chellappa, U. Akdemir, L. Davis, An ontology of video events in the physical security and surveillance domain, online, http://www.ai.sri.com/ burns/EventOntology (2003).

[39] R. Nevatia, T. Zhao, S. Hongeng, Hierarchical language-based representation of events in video streams, in: Procs. of the IEEE Workshop on Event Mining, 2003.

[40] R. Romdhane, B. Boulay, F. Bremond, M. Thonnat, Probabilistic recognition of complex event, in: Procs. of ICCVS, 2011, pp. 122-131.

[41] A. Hakeem, M. Shah, Learning, detection and representation of multi-agent events in videos, Artif. Intell. 171 (89) (2007) 586-605.

[42] S. Khokhar, I. Saleemi, M. Shah, Multi-agent event recognition by preservation of spatiotemporal relationships between probabilistic models, Image Vision Comput. 31 (9) (2013) 603-615.

[43] S. D. Tran, L. S. Davis, Event modeling and recognition using markov logic networks, in: Procs. of ECCV, 2008, pp. 610-623.

[44] A. Stolcke, An efficient probabilistic context-free parsing algorithm that computes prefix probabilities, in: Computational Linguistics, MIT Press for the Association for Computational Linguistics, 1995.

[45] Y. Ivanov, A. Bobick, Recognition of visual activities and interactions by stochastic parsing, IEEE Transactions on Pattern Analysis and Machine Intelligence 22 (8) (2000) 852-872.

[46] A. Kanaujia, T. Choe, H. Deng, Complex events recognition under uncertainty in a sensor network (2014). doi:arXiv:1411.0085.

[47] W. Brendel, A. Fern, S. Todorovic, Probabilistic event logic for interval-based event recognition., in: Procs. of 
CVPR, 2011, pp. 3329-3336.

[48] A. Dempster, Upper and lower probabilities induced by a multivalued mapping, The Annals of Statistics 28 (1967) 325-339.

[49] G. Shafer, A Mathematical Theory of Evidence, Princeton University Press, 1976.

[50] J. Allen, Maintaining knowledge about temporal intervals, Communications of the ACM 26 (11) (1983) 832843.

[51] W. Liu, J. Hughes, M. McTear, Representing heuristic knowledge in the DS theory, in: Procs. of UAI, 1992, pp. 182-190.

[52] J. Lowrance, T. Garvey, T. Strat, A framework for evidential-reasoning systems, in: Procs. of AAAI, 1986, pp. 896-903.

[53] P. Smets, Constructing the pignistic probability function in a context of uncertainty, in: Procs. of UAI, 1990, pp. $29-40$.

[54] H. Xu, Y. Hsia, P. Smets, Transferable belief model for decision making in the valuation-based systems, IEEE Transactions on Systems, Man, and Cybernetics-Part A: System and Humans 26 (6) (1996) 698-707.

[55] J. Allen, An interval-based representation of temporal knowledge, in: Proc. of IJCAI, 1981, pp. 221-225.

[56] N. McLaughlin, J. Martinez-del Rincon, P. Miller, Online multiperson tracking with occlusion reasoning and unsupervised track motion model, in: Procs. of AVSS, 2013, pp. 37-42.

[57] X. Hong, Y. Huang, W. Ma, P. Miller, W. Liu, H. Zhou, Video event recognition by Dempster-Shafer theory, in: Procs. of ECAI, 2014.

[58] X. Hong, W. Ma, Y. Huang, P. Miller, W. Liu, H. Zhou, Evidence reasoning for event inference in smart transport video surveillance, in: Procs. of ICDSC, 2014.

[59] J. Ma, W. Liu, P. Miller, W. Yan, Event composition with imperfect information for bus surveillance, in: Procs. of AVSS, 2009, pp. 382-387. 